\title{
Multiplication in Sobolev spaces, revisited
}

\author{
A. Behzadan and M. Holst
}

\begin{abstract}
In this article, we re-examine some of the classical pointwise multiplication theorems in Sobolev-Slobodeckij spaces, in part motivated by a simple counter-example that illustrates how certain multiplication theorems fail in Sobolev-Slobodeckij spaces when a bounded domain is replaced by $\mathbb{R}^{n}$. We identify the source of the failure, and examine why the same failure is not encountered in Bessel potential spaces. To analyze the situation, we begin with a survey of the classical multiplication results stated and proved in the 1977 article of Zolesio, and carefully distinguish between the case of spaces defined on the all of $\mathbb{R}^{n}$ and spaces defined on a bounded domain (with e.g. a Lipschitz boundary). However, the survey we give has a few new wrinkles; the proofs we include are based almost exclusively on interpolation theory rather than LittlewoodPaley theory and Besov spaces, and some of the results we give and their proofs, including the results for negative exponents, do not appear in the literature in this form. We also include a particularly important variation of one of the multiplication theorems that is relevant to the study of nonlinear PDE systems arising in general relativity and other areas. The conditions for multiplication to be continuous in the case of Sobolev-Slobodeckij spaces are somewhat subtle and intertwined, and as a result, the multiplication theorems of Zolesio in 1977 have been cited (more than once) in the standard literature in slightly more generality than what is actually proved by Zolesio, and in cases that allow for construction of counter-examples such as the one included here.
\end{abstract}

\section{Contents}

1 Introduction . . . . . . . . . . . . . . . . . . . . . . . . . . . 276

2 Notation and definitions . . . . . . . . . . . . . . . . . . . . . . . . . 277

3 Key properties of Sobolev spaces . . . . . . . . . . . . . . . . . . . . . . . . . . . . . . 281

4 A counter-example for generalized Holder-type inequalities in $W^{s, p}$. . . . . . . . . . . . 284

5 Sufficient conditions for $H^{s_{1}, p_{1}} \times H^{s_{2}, p_{2}} \hookrightarrow H^{s, p}, s \geq 0, s \in \mathbb{R}$. . . . . . . . . . . . . . . 286

6 Sufficient conditions for $W^{s_{1}, p_{1}} \times W^{s_{2}, p_{2} \hookrightarrow W^{s, p}, s \geq 0, s \in \mathbb{N}_{0}}$. . . . . . . . . . . . . . . 289

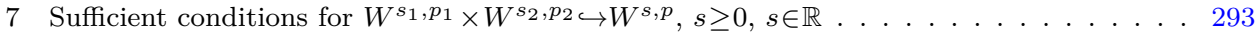

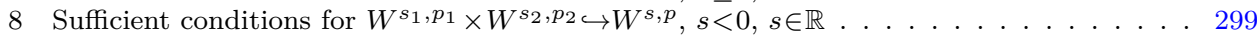

A A useful version of the multiplication theorem . . . . . . . . . . . . . . . . . . 303

References . . . . . . . . . . . . . . . . . . . . . 305

Key words and phrases: Sobolev spaces, Bessel potential spaces, Triebel-Lizorkin spaces, multiplication, real interpolation, complex interpolation. 


\section{Introduction}

Let $f \in W^{s_{1}, p_{1}}$ and $g \in W^{s_{2}, p_{2}}$. What can be said about the product $f g$ ? In particular, to which Sobolev space $W^{s, p}$ does the product $f g$ belong? The answer to this question plays a key role in a number of applications in analysis, and having a very complete answer to this question is critical to the modern theory of partial differential equations (PDE). It is particularly important to the understanding of the solution theory for elliptic PDE, and it is also central to the design and analysis of approximations of their solutions using various techniques. In the modern theory of partial differential equations, PDE are interpreted as equations of the form $A u=f$ where $A$ is an operator between suitable function spaces. In this view, the existence of a unique solution for all right hand sides is equivalent to $A$ being bijective. A main difficulty is in choosing the domain of realization and the codomain of the operator $A$, that is, choosing appropriate function spaces $X$ and $Y$ such that

(1) $A$ can be considered as an operator from $X$ to $Y$ and $f \in Y$, i.e., we need to ensure that the equation makes sense if we consider $X$ and $Y$ as the domain and codomain of $A$.

(2) $A$ (or a family of approximations of $A$ ) has "nice" properties as an operator (or a family of operators) from $X$ to $Y$. Here "nice properties" may refer to any of the following: $A$ is continuous, $A$ is compact, $A$ is Fredholm, $A$ is injective, $A$ is surjective, $A$ satisfies a maximum principle, etc.

As it turns out, for elliptic equations, using Sobolev spaces (or weighted Sobolev spaces) as domain and codomain of $A$ helps us to ensure that $A$ has "nice" properties. But how to determine appropriate Sobolev spaces to make sure that the equation makes sense? This is one of the applications where pointwise multiplication theorems are particularly important. The best way to see this is by looking at a very simple example. Consider the equation $-\Delta u+V u=f$ in $\Omega \subseteq \mathbb{R}^{n}$ where $\Omega$ is a domain with smooth boundary. Suppose we want to seek the unknown function $u$ in the Sobolev space $W^{s, p}$ where $s \geq 2$ and $1<p<\infty$. Having this assumption, what restrictions do we need to impose on the data $V$ and $f$ ? The assumption $u \in W^{s, p}$ implies that $-\Delta u \in W^{s-2, p}$. Therefore for the equation to make sense (as an equality in $\left.W^{s-2, p}\right), f$ and $V u$ must belong to $W^{s-2, p}$. So now we need to find those Sobolev spaces $W^{r, q}$ such that if $V \in W^{r, q}$, then $V u \in W^{s-2, p}$. That is, we need to find those exponents $r$ and $q$ for which the product of a function in $W^{r, q}$ and a function in $W^{s, p}$ belongs to $W^{s-2, p}$. If one now considers even the simplest nonlinear generalization of this problem, say $-\Delta u+V u^{p}=f$ in $\Omega \subseteq \mathbb{R}^{n}$, then it is immediately clear that the conditions on the spaces become substantially more complicated, and multiplication theorems are a critical tool in the analysis of nonlinear PDE. 
There are a number of articles and book chapters that are devoted to the study of pointwise multiplication in function spaces, e.g. [9] and [12]. Unfortunately most references study the question in the general setting of Triebel-Lizorkin spaces and use technical tools from Littlewood-Paley theory and theory of Besov spaces to prove the results. A main feature of this article is that the key results are proved without any direct reference to Littlewood-Paley theory and Besov spaces, which makes it accessible to a wider range of readers. In particular, we give alternative proofs for a number of results first stated in [12] for Sobolev spaces with nonnegative exponents. Additionally, we extend those results to Sobolev spaces with negative exponents. We clearly distinguish between the case of Sobolev spaces defined on the entire space $\mathbb{R}^{n}$ and the case where Sobolev spaces are defined on a bounded domain. Lastly, we remark that one of the main tools we use throughout the paper, namely interpolation theory, is a fascinating topic itself; we only briefly summarize some of the main ideas and results we need in the paper in Section 3. Much more complete expositions can be found, for example, in [11].

\section{Outline of the paper}

A brief outline of the remainder of the paper is as follows. In Section 2 we review some of the basic well-known definitions and facts about Sobolev spaces. In Section 3 we summarize some basic facts about interpolation theory and several key properties of Sobolev spaces. In Section 4 we review a counter-example for generalized Holder-type inequalities in Sobolev-Slobodeckij spaces. In Sections 5, 6,7 , and 8 we state and prove the main theorems.

\section{Notation and definitions}

In this section we briefly review some basic notation and definitions related to the Sobolev spaces, with emphasis on fractional order spaces. Throughout the manuscript we use the notation $A \preceq B$ to mean $A \leq c B$, where $c$ is a positive constant that does not depend on the non-fixed parameters appearing in $A$ and $B$. We use the notation $X \hookrightarrow Y$ to mean $X \subseteq Y$ and the inclusion map is continuous.

Definition 2.1. Let $k \in \mathbb{N}_{0}, 1<p<\infty$. The Sobolev space $W^{k, p}\left(\mathbb{R}^{n}\right)$ is defined as follows:

$$
W^{k, p}\left(\mathbb{R}^{n}\right)=\left\{u \in L^{p}\left(\mathbb{R}^{n}\right):\|u\|_{W^{k, p}\left(\mathbb{R}^{n}\right)}:=\sum_{|\nu| \leq k}\left\|\partial^{\nu} u\right\|_{p}<\infty\right\} .
$$

For $k \in \mathbb{N}$, the Sobolev space $W^{-k, p}\left(\mathbb{R}^{n}\right)$ is defined as the topological dual of $W^{k, p^{\prime}}\left(\mathbb{R}^{n}\right)$ where $\frac{1}{p}+\frac{1}{p^{\prime}}=1$. That is, $W^{-k, p}\left(\mathbb{R}^{n}\right):=\left(W^{k, p^{\prime}}\left(\mathbb{R}^{n}\right)\right)^{*}$. 
Remark 2.2.

- For real-valued function $u\left(x_{1}, \ldots, x_{n}\right)$ and multi-index $\nu=\left(\nu_{1}, \ldots, \nu_{n}\right) \in \mathbb{N}_{0}^{n}$,

$$
|\nu|:=\nu_{1}+\ldots+\nu_{n}, \quad \partial^{\nu} u:=\frac{\partial^{|\nu|} u}{\partial x_{1}^{\nu_{1}} \ldots \partial x_{n}^{\nu_{n}}}, \quad\|u\|_{p}:=\left(\int_{\mathbb{R}^{n}}|u|^{p} d x\right)^{\frac{1}{p}} .
$$

(Note that $\partial^{\nu} u$ in Definition 2.1 should be interpreted in the weak sense as explained for example in [1].)

- The Sobolev norm is defined so that $\partial^{\alpha}: W^{k, p}\left(\mathbb{R}^{n}\right) \rightarrow W^{k-|\alpha|, p}\left(\mathbb{R}^{n}\right)$ becomes a continuous operator for $|\alpha| \leq k$. It can be shown that $C_{c}^{\infty}\left(\mathbb{R}^{n}\right)$ is dense in $W^{k, p}\left(\mathbb{R}^{n}\right)$. In fact, $W^{k, p}\left(\mathbb{R}^{n}\right)$ is the completion of the space of smooth functions with respect to $\|\cdot\|_{W^{k, p}\left(\mathbb{R}^{n}\right)}$.

- Clearly, if $k_{1} \geq k_{0}$, then $W^{k_{1}, p}\left(\mathbb{R}^{n}\right) \subseteq W^{k_{0}, p}\left(\mathbb{R}^{n}\right)$.

There are nonequivalent ways to generalize the above definition to allow noninteger exponents. We can define Sobolev spaces with noninteger exponents as

(1) Slobodeckij spaces, or,

(2) Bessel potential spaces.

There are at least three equivalent methods to define each of the above spaces:

(1) Classical definition

(2) Definition based on interpolation theory

(3) Definition based on Littlewood-Paley theory

\section{1-Classical definitions}

Definition 2.3. Let $s \in \mathbb{R}$ and $p \in[1, \infty]$. The Sobolev-Slobodeckij space $W^{s, p}\left(\mathbb{R}^{n}\right)$ is defined as follows:

- If $s=k \in \mathbb{N}_{0}, p \in[1, \infty]$,

$$
W^{k, p}\left(\mathbb{R}^{n}\right)=\left\{u \in L^{p}\left(\mathbb{R}^{n}\right):\|u\|_{W^{k, p}\left(\mathbb{R}^{n}\right)}:=\sum_{|\nu| \leq k}\left\|\partial^{\nu} u\right\|_{p}<\infty\right\} .
$$

- If $s=\theta \in(0,1), p \in[1, \infty)$,

$$
\begin{aligned}
& W^{\theta, p}\left(\mathbb{R}^{n}\right)=\left\{u \in L^{p}\left(\mathbb{R}^{n}\right):|u|_{W^{\theta, p}\left(\mathbb{R}^{n}\right)}:=\left(\iint_{\mathbb{R}^{n} \times \mathbb{R}^{n}} \frac{|u(x)-u(y)|^{p}}{|x-y|^{n+\theta p}} d x d y\right)^{\frac{1}{p}}<\infty\right\} . \\
& \bullet \text { If } s=\theta \in(0,1), p=\infty, \\
& W^{\theta, \infty}\left(\mathbb{R}^{n}\right)=\left\{u \in L^{\infty}\left(\mathbb{R}^{n}\right):|u|_{W^{\theta, \infty\left(\mathbb{R}^{n}\right)}}:=\operatorname{ess}_{x, y \in \mathbb{R}^{n}, x \neq y} \frac{|u(x)-u(y)|}{|x-y|^{\theta}}<\infty\right\} .
\end{aligned}
$$


- If $s=k+\theta, k \in \mathbb{N}_{0}, \theta \in(0,1), p \in[1, \infty]$, $W^{s, p}\left(\mathbb{R}^{n}\right)=\left\{u \in W^{k, p}\left(\mathbb{R}^{n}\right):\|u\|_{W^{s, p}\left(\mathbb{R}^{n}\right)}:=\|u\|_{W^{k, p}\left(\mathbb{R}^{n}\right)}+\sum_{|\nu|=k}\left|\partial^{\nu} u\right|_{W^{\theta, p}\left(\mathbb{R}^{n}\right)}<\infty\right\}$.

- If $s<0$ and $p \in(1, \infty)$,

$$
W^{s, p}\left(\mathbb{R}^{n}\right)=\left(W^{-s, p^{\prime}}\left(\mathbb{R}^{n}\right)\right)^{*} \quad\left(\frac{1}{p}+\frac{1}{p^{\prime}}=1\right) .
$$

Alternatively, for $s \in \mathbb{R}$ and $1<p<\infty$, one can define Sobolev spaces as Bessel potential spaces $H^{s, p}\left(\mathbb{R}^{n}\right)$ :

$$
H^{s, p}\left(\mathbb{R}^{n}\right)=\left\{u \in S^{\prime}\left(\mathbb{R}^{n}\right):\|u\|_{W^{s, p}\left(\mathbb{R}^{n}\right)}:=\left\|\mathcal{F}^{-1}\left(\langle\xi\rangle^{s} \mathcal{F} u\right)\right\|_{L^{p}}<\infty\right\},
$$

where $\langle\xi\rangle:=\left(1+|\xi|^{2}\right)^{\frac{1}{2}}$. Here $\mathcal{F}$ denotes the Fourier transform on the space $S^{\prime}\left(\mathbb{R}^{n}\right)$ of tempered distributions. It is a well-known fact that $H^{s, p}\left(\mathbb{R}^{n}\right)=\left(H^{-s, p^{\prime}}\left(\mathbb{R}^{n}\right)\right)^{*}$ and for $k \in \mathbb{Z}$ the two definitions agree (see e.g. [6], [10] and [11]). Also, for $s \in \mathbb{R}$ and $p=2$ the two definitions agree (see e.g. [6] and [10]). $H^{s, 2}\left(\mathbb{R}^{n}\right)$ is often denoted by $H^{s}\left(\mathbb{R}^{n}\right)$.

\section{2-definitions based on interpolation theory}

A short introduction to interpolation theory in Banach spaces is given in Section 3. Suppose $s \in \mathbb{R} \backslash \mathbb{Z}, 1<p<\infty$, and let $\theta:=s-\lfloor s\rfloor$.

- $W^{s, p}\left(\mathbb{R}^{n}\right)=\left(W^{\lfloor s\rfloor, p}\left(\mathbb{R}^{n}\right), W^{\lfloor s\rfloor+1, p}\left(\mathbb{R}^{n}\right)\right)_{\theta, p}$.

- $H^{s, p}\left(\mathbb{R}^{n}\right)=\left[H^{\lfloor s\rfloor, p}\left(\mathbb{R}^{n}\right), H^{\lfloor s\rfloor+1, p}\left(\mathbb{R}^{n}\right)\right]_{\theta}$.

\section{3-definitions based on Littlewood-Paley theory}

Consider an open cover of $\mathbb{R}^{n}$ that consists of the following sets (annuli):

$$
B_{2}, \quad B_{4} \backslash \overline{B_{1}}, \quad B_{8} \backslash \overline{B_{2}}, \quad \ldots, \quad B_{2^{j+1}} \backslash \bar{B}_{2^{j-1}},
$$

where $B_{r}$ is the open ball of radius $r$ centered at the origin. Consider the following smooth partition of unity subordinate to the above cover of $\mathbb{R}^{n}$ :

$$
\begin{aligned}
& \varphi_{0}=1 \quad \text { on } B_{1}, \operatorname{supp} \varphi_{0} \subseteq B_{2}, \\
& \varphi(\xi)=\varphi_{0}(\xi)-\varphi_{0}(2 \xi) \quad\left(\operatorname{supp} \varphi \subseteq B_{2}, \varphi=0 \text { on } B_{\frac{1}{2}}\right), \\
& \forall j \geq 1 \quad \varphi_{j}(\xi)=\varphi\left(2^{-j} \xi\right) .
\end{aligned}
$$

One can easily check that $\sum_{j=0}^{\infty} \varphi_{j}(\xi)=1$. 
Definition 2.4 .

- For $s \in \mathbb{R}, 1 \leq p<\infty$, and $1 \leq q<\infty$ (or $p=q=\infty$ ) we define the Triebel-Lizorkin space $F_{p, q}^{s}\left(\mathbb{R}^{n}\right)$ as follows

$$
F_{p, q}^{s}\left(\mathbb{R}^{n}\right)=\left\{u \in S^{\prime}\left(\mathbb{R}^{n}\right):\|u\|_{F_{p, q}^{s}\left(\mathbb{R}^{n}\right)}=\|\| 2^{s j} \mathcal{F}^{-1}\left(\varphi_{j} \mathcal{F} u\right)\left\|_{l^{q}}\right\|_{L^{p}\left(\mathbb{R}^{n}\right)}<\infty\right\} .
$$

Note that, in particular, for $1 \leq p, q<\infty$ we have

$$
\|u\|_{F_{p, q}^{s}\left(\mathbb{R}^{n}\right)}=\left(\int_{\mathbb{R}^{n}}\left(\sum_{j=0}^{\infty}\left(2^{s j}\left|\mathcal{F}^{-1}\left(\varphi_{j} \mathcal{F} u\right)\right|\right)^{q}\right)^{\frac{p}{q}} d x\right)^{\frac{1}{p}} .
$$

- For $s \in \mathbb{R}, 1 \leq p<\infty$, and $1 \leq q<\infty$ we define the Besov space $B_{p, q}^{s}\left(\mathbb{R}^{n}\right)$ as follows

$$
B_{p, q}^{s}\left(\mathbb{R}^{n}\right)=\left\{u \in S^{\prime}\left(\mathbb{R}^{n}\right):\|u\|_{B_{p, q}^{s}\left(\mathbb{R}^{n}\right)}=\|\| 2^{s j} \mathcal{F}^{-1}\left(\varphi_{j} \mathcal{F} u\right)\left\|_{L^{p}\left(\mathbb{R}^{n}\right)}\right\|_{l^{q}}<\infty\right\} .
$$

Note that we have

$$
\|u\|_{B_{p, q}^{s}\left(\mathbb{R}^{n}\right)}=\left(\sum_{j=0}^{\infty}\left(\int_{\mathbb{R}^{n}}\left(2^{s j}\left|\mathcal{F}^{-1}\left(\varphi_{j} \mathcal{F} u\right)\right|\right)^{p}\right)^{\frac{q}{p}}\right)^{\frac{1}{q}} .
$$

We have the following relations (see e.g. [5], [10] and [11])

- $L^{p}=F_{p, 2}^{0}, 1<p<\infty$.

- $B_{p, p}^{s}=F_{p, p}^{s}, s \in \mathbb{R}, 1<p<\infty$.

- $H^{s, p}=F_{p, 2}^{s}, s \in \mathbb{R}, 1<p<\infty$.

- $W^{k, p}=H^{k, p}=F_{p, 2}^{k}, k \in \mathbb{Z}, 1<p<\infty$.

- $W^{s, p}=B_{p, p}^{s}=F_{p, p}^{s}, s \in \mathbb{R} \backslash \mathbb{Z}, 1<p<\infty$.

- If $k \in \mathbb{N}$, then $B_{p, p}^{k} \hookrightarrow W^{k, p}$ for $1 \leq p \leq 2$ and $W^{k, p} \hookrightarrow B_{p, p}^{k}$ for $p \geq 2$.

Definition 2.5. Let $\Omega$ be an open bounded subset of $\mathbb{R}^{n}$ with Lipschitz continuous boundary. Suppose $s \geq 0$ and $1 \leq p<\infty$. $W^{s, p}(\Omega)$ is defined as the restriction of $W^{s, p}\left(\mathbb{R}^{n}\right)$ to $\Omega$ and is equipped with the following norm:

$$
\|u\|_{W^{s, p}(\Omega)}=\inf _{v \in W^{s, p}\left(\mathbb{R}^{n}\right),\left.v\right|_{\Omega}=u}\|v\|_{W^{s, p}\left(\mathbb{R}^{n}\right)} .
$$

$W_{0}^{s, p}(\Omega)$ is defined as the closure of $C_{c}^{\infty}(\Omega)$ in $W^{s, p}(\Omega) . W_{0}^{s, 2}(\Omega)$ is often denoted by $\stackrel{\circ}{H}^{s}(\Omega)$.

Remark 2.6.

- One may define $H^{s, p}(\Omega), B_{p, q}^{s}(\Omega)$, and $F_{p, q}^{s}(\Omega)$ in a similar fashion. 
- It can be shown that for $k \in \mathbb{N}_{0}$ and $1<p<\infty$ the above definition of $W^{k, p}(\Omega)$ agrees with the following intrinsic definition (see e.g. [11])

$$
W^{k, p}(\Omega)=\left\{u \in L^{p}(\Omega):\|u\|_{W^{k, p}(\Omega)}:=\sum_{|\nu| \leq k}\left\|\partial^{\nu} u\right\|_{L^{p}(\Omega)}<\infty\right\} .
$$

Indeed, for all $s>0$ and $1<p<\infty$, the Sobolev space $W^{s, p}(\Omega)$ can be equivalently defined using intrinsic norms analogous to those displayed in Definition 2.3.

For $s<0$ and $1<p<\infty$ we define $W^{s, p}(\Omega):=\left(W_{0}^{-s, p^{\prime}}(\Omega)\right)^{*}$.

When there is no danger of ambiguity about the domain we may write

- $W^{s, p}$ instead of $W^{s, p}(\Omega)$,

- $\|\cdot\|_{W^{s, p}}$ or $\|\cdot\|_{s, p}$ instead of $\|\cdot\|_{W^{s, p}(\Omega)}$.

\section{Key properties of Sobolev spaces}

We begin with reviewing the basic definitions of interpolation theory in Banach spaces. A detailed discussion can be found in [11].

A pair $\left\{A_{0}, A_{1}\right\}$ of two Banach spaces is said to be an interpolation couple, if both spaces are continuously embedded in a common Hausdorff topological vector space $A$. We may consider the following two subspaces:

- $A_{0} \cap A_{1}$, and

- $A_{0}+A_{1}:=\left\{a \in A: \exists a_{0} \in A_{0}, \exists a_{1} \in A_{1}, a=a_{0}+a_{1}\right\}$.

Equipped with the norms

$$
\begin{aligned}
& \|a\|_{A_{0} \cap A_{1}}:=\min \left\{\|a\|_{A_{0}},\|a\|_{A_{1}}\right\} \\
& \|a\|_{A_{0}+A_{1}}:=\inf \left\{\left\|a_{0}\right\|_{A_{0}}+\left\|a_{1}\right\|_{A_{1}}: a=a_{0}+a_{1}\right\}
\end{aligned}
$$

$A_{0} \cap A_{1}$ and $A_{0}+A_{1}$ become Banach spaces. Real interpolation and complex interpolation are two, generally nonequivalent, methods for constructing intermediate spaces between $A_{0}$ and $A_{1}$ in the sense that the new space lies between $A_{0} \cap A_{1}$ and $A_{0}+A_{1}$ (with continuous injections).

- Given a pair $(\theta, p)$ with $0<\theta<1$ and $1<p<\infty$, the real interpolation functor constructs an intermediate Banach space denoted by $\left(A_{0}, A_{1}\right)_{\theta, p}$.

- Given $0<\theta<1$, the complex interpolation functor constructs an intermediate Banach space denoted by $\left[A_{0}, A_{1}\right]_{\theta}$.

Theorem 3.1. (Real Interpolation) [11] Let $\Omega$ be a bounded open set with smooth boundary in $\mathbb{R}^{n}$ or $\Omega=\mathbb{R}^{n}$. Suppose $\theta \in(0,1), 0 \leq s_{0}, s_{1}<\infty$, and $1<p_{0}, p_{1}<$ $\infty$. Additionally assume one of the following cases holds: 
- $s_{0}, s_{1}, s$ are nonintegers.

- $s_{0} \in \mathbb{R}, s_{1} \in \mathbb{Z}$, and $s \in \mathbb{R} \backslash \mathbb{Z}$.

If

$$
s=(1-\theta) s_{0}+\theta s_{1}, \quad \frac{1}{p}=\frac{1-\theta}{p_{0}}+\frac{\theta}{p_{1}},
$$

then $W^{s, p}(\Omega)=\left(W^{s_{0}, p_{0}}(\Omega), W^{s_{1}, p_{1}}(\Omega)\right)_{\theta, p}$.

Theorem 3.2. (Complex Interpolation) [11] Let $\Omega$ be a bounded open set with smooth boundary in $\mathbb{R}^{n}$ or $\Omega=\mathbb{R}^{n}$. Suppose $\theta \in(0,1), 0 \leq s_{0}, s_{1}<\infty$, and $1<p_{0}, p_{1}<$ $\infty$. If

$$
s=(1-\theta) s_{0}+\theta s_{1}, \quad \frac{1}{p}=\frac{1-\theta}{p_{0}}+\frac{\theta}{p_{1}},
$$

then

- $H^{s, p}(\Omega)=\left[H^{s_{0}, p_{0}}(\Omega), H^{s_{1}, p_{1}}(\Omega)\right]_{\theta}$.

- $W^{s, p}(\Omega)=\left[W^{s_{0}, p_{0}}(\Omega), W^{s_{1}, p_{1}}(\Omega)\right]_{\theta}$ provided $s_{0}, s_{1}, s>0$ are nonintegers.

- $W^{s, p}(\Omega) \hookrightarrow\left[W^{s_{0}, p_{0}}(\Omega), W^{s_{1}, p_{1}}(\Omega)\right]_{\theta}$ provided $s_{0}$ and $s_{1}$ are not integers and $p \geq 2$.

(This is a consequence of the fact that for $s_{0}, s_{1} \notin \mathbb{Z},\left[W^{s_{0}, p_{0}}(\Omega), W^{s_{1}, p_{1}}(\Omega)\right]_{\theta}=$ $B_{p, p}^{s}$. If $s \notin \mathbb{Z}$, then $B_{p, p}^{s}=W^{s, p} ;$ if $s \in \mathbb{Z}$, then $W^{s, p} \hookrightarrow B_{p, p}^{s}$ provided $p \geq 2$.)

Remark 3.3. According to [11], the above interpolation facts remain true even if we only assume the bounded open set $\Omega$ is of cone-type. According to [1] if $\Omega$ is a bounded open set with Lipschitz continuous boundary, then it is of cone-type.

Theorem 3.4. (Properties of the Spaces $\left.\left(A_{0}, A_{1}\right)_{\theta, p}\right)[11]$

- It holds that $\left(A_{0}, A_{1}\right)_{\theta, p}=\left(A_{1}, A_{0}\right)_{1-\theta, p}$.

- If $A_{0} \hookrightarrow A_{1}$, then for $0<\theta<\tilde{\theta}<1$ and $1<p, \tilde{p}<\infty$

$$
\left(A_{0}, A_{1}\right)_{\theta, p} \longleftrightarrow\left(A_{0}, A_{1}\right)_{\tilde{\theta}, \tilde{p}}
$$

Theorem 3.5. (Interpolation Properties of Linear Operators) [9] Let $A_{0} \subseteq A_{1}$ and $B_{0} \subseteq B_{1}$ be couples of Banach spaces. If $T_{1}: A_{1} \rightarrow B_{1}$ is a continuous linear map that restricts to a continuous linear map $T_{0}: A_{0} \rightarrow B_{0}$, then $T_{1}$ also restricts to a continuous linear map from $\left(A_{0}, A_{1}\right)_{\theta, p}$ to $\left(B_{0}, B_{1}\right)_{\theta, p}$ for all $0<\theta<1$ and $1<p<\infty$.

Theorem 3.6. (Interpolation Properties of Bilinear Maps) [11] Let $A_{0} \subseteq A_{1}$, $B_{0} \subseteq B_{1}$, and $C_{0} \subseteq C_{1}$ be couples of Banach spaces. If $T_{1}: A_{1} \times B_{1} \rightarrow C_{1}$ is a continuous bilinear map that restricts to a continuous bilinear map $T_{0}: A_{0} \times B_{0} \rightarrow C_{0}$, then $T_{1}$ also restricts to a continuous bilinear map

- (complex interpolation) from $\left[A_{0}, A_{1}\right]_{\theta} \times\left[B_{0}, B_{1}\right]_{\theta}$ into $\left[C_{0}, C_{1}\right]_{\theta}$, and

- (real interpolation) from $\left(A_{0}, A_{1}\right)_{\theta, p} \times\left(B_{0}, B_{1}\right)_{\theta, q}$ into $\left(C_{0}, C_{1}\right)_{\theta, r}$ where $0<\theta<1$ and $\frac{1}{r}=\frac{1}{p}+\frac{1}{q}-1 \geq 0$. 
Theorem 3.7. (Extension Property) [4] Let $\Omega \subset \mathbb{R}^{n}$ be a bounded open set with Lipschitz continuous boundary. Then for all $s>0$ and for $1 \leq p<\infty$, there exists a continuous linear extension operator $P: W^{s, p}(\Omega) \hookrightarrow W^{s, p}\left(\mathbb{R}^{n}\right)$ such that $\left.(P u)\right|_{\Omega}=u$ and $\|P u\|_{W^{s, p}\left(\mathbb{R}^{n}\right)} \leq C\|u\|_{W^{s, p}(\Omega)}$ for some constant $C$ that may depend on $s, p$, and $\Omega$ but is independent of $u$.

Theorem 3.8. (Embedding Theorem I) (see e.g. [4], [11] and [12]) Let $\Omega$ be a bounded open subset of $\mathbb{R}^{n}$ with Lipschitz continuous boundary or $\Omega=\mathbb{R}^{n}$. Suppose $1 \leq p \leq q<\infty$ and $0 \leq t \leq s$ satisfy $s-\frac{n}{p} \geq t-\frac{n}{q}$. Then

- $W^{s, p}(\Omega) \hookrightarrow W^{t, q}(\Omega)$,

- $H^{s, p}\left(\mathbb{R}^{n}\right) \hookrightarrow H^{t, q}\left(\mathbb{R}^{n}\right)$ provided we assume $p>1$.

Theorem 3.9. (Embedding Theorem II) [7] Let $\Omega$ be a bounded open subset of $\mathbb{R}^{n}$ with Lipschitz continuous boundary or $\Omega=\mathbb{R}^{n}$.

(i) If $s p>n$, then $W^{s, p}(\Omega) \hookrightarrow C^{0}(\bar{\Omega})$ and $W^{s, p}(\Omega)$ is a Banach algebra.

(ii) If $s p=n$, then $W^{s, p}(\Omega) \hookrightarrow L^{q}(\Omega)$ for $p \leq q<\infty$.

(iii) If $0 \leq s p<n$, then $W^{s, p}(\Omega) \hookrightarrow L^{q}(\Omega)$ for $p \leq q \leq p^{*}=\frac{n p}{n-s p}$.

(Items (ii) and (iii) are direct consequences of Theorem 3.8.) The following result is a generalization of the well-known embedding relationships; a simple proof does not appear to be in the literature, so we include the short proof.

Theorem 3.10. (Embedding Theorem III) Let $\Omega$ be a bounded open subset of $\mathbb{R}^{n}$ with Lipschitz continuous boundary. Suppose $1 \leq p, q<\infty$ ( $p$ does NOT need to be less than $q$ ) and $0 \leq t \leq s$ satisfy $s-\frac{n}{p} \geq t-\frac{n}{q}$. If $s \notin \mathbb{N}_{0}$, additionally assume that $s \neq t$. Then $W^{s, p}(\Omega) \hookrightarrow W^{t, q}(\Omega)$.

Proof of Theorem 3.10. If $p \leq q$, the claim follows from Theorem 3.8. So we may assume $p>q$. We consider four cases:

Case $1 s=t=k \in \mathbb{N}_{0}$ : Note that since $\Omega$ is a bounded open set, $L^{p}(\Omega) \hookrightarrow L^{q}(\Omega)$. We can write

$$
\|u\|_{W^{k, q}(\Omega)}=\sum_{|\beta| \leq k}\left\|\partial^{\beta} u\right\|_{L^{q}(\Omega)} \preceq \sum_{|\beta| \leq k}\left\|\partial^{\beta} u\right\|_{L^{p}(\Omega)}=\|u\|_{W^{k, p}(\Omega)} .
$$

which precisely means that $W^{k, p}(\Omega) \hookrightarrow W^{k, q}(\Omega)$.

Case $2 \exists k \in \mathbb{N}_{0}$ such that $t=k<s$ : It follows from Theorem 3.8 that $W^{s, p}(\Omega) \hookrightarrow W^{k, p}(\Omega)$. Now notice that, by what was proved in Case 1, $W^{k, p}(\Omega) \hookrightarrow W^{k, q}(\Omega)$. 
Case $3 \exists k \in \mathbb{N}_{0}$ such that $k<t<s<k+1$ (and, as assumed $p>q$ ): Let

$$
\begin{aligned}
& \theta=s-\lfloor s\rfloor \quad(\text { so } s=(1-\theta) k+\theta(k+1)), \\
& \tilde{\theta}=t-\lfloor t\rfloor \quad(\text { so } t=(1-\tilde{\theta}) k+\tilde{\theta}(k+1)) .
\end{aligned}
$$

Note that since $t<s$, we have $\tilde{\theta}<\theta$. By what was shown in Case 1:

$$
W^{k, p}(\Omega) \longleftrightarrow W^{k, q}(\Omega), \quad W^{k+1, p}(\Omega) \longleftrightarrow W^{k+1, q}(\Omega) .
$$

Since $s=(1-\theta) k+\theta(k+1)$, it follows from interpolation properties of linear operators (Theorem 3.5) that

$$
\begin{gathered}
W^{s, p}(\Omega)=\left(W^{k, p}(\Omega), W^{k+1, p}(\Omega)\right)_{\theta, p} \longleftrightarrow\left(W^{k, q}(\Omega), W^{k+1, q}(\Omega)\right)_{\theta, p} \\
\stackrel{\text { Theorem }}{=}{ }^{3.4}\left(W^{k+1, q}(\Omega), W^{k, q}(\Omega)\right)_{1-\theta, p} .
\end{gathered}
$$

Since $1-\theta<1-\tilde{\theta}$ and $W^{k+1, q_{\hookrightarrow}} W^{k, q}$, it follows from Theorem 3.4 that

$$
\begin{aligned}
\left(W^{k+1, q}(\Omega), W^{k, q}(\Omega)\right)_{1-\theta, p} & \longleftrightarrow\left(W^{k+1, q}(\Omega), W^{k, q}(\Omega)\right)_{1-\tilde{\theta}, q} \\
& \longleftrightarrow\left(W^{k, q}(\Omega), W^{k+1, q}(\Omega)\right)_{\tilde{\theta}, q}=W^{t, q}(\Omega) .
\end{aligned}
$$

Thus $W^{s, p}(\Omega) \hookrightarrow W^{t, q}(\Omega)$ as desired.

Case $4 \exists k \in \mathbb{N}$ such that $t<k<s$ (of course $p>q$ ): Let $\hat{s}$ be a number in the open interval $(t,\lfloor t\rfloor+1)$. It follows from Theorem 3.8 that $W^{s, p}(\Omega) \hookrightarrow W^{\hat{s}, p}(\Omega)$. Now notice that, by what was proved in Case $3, W^{\hat{s}, p}(\Omega) \hookrightarrow W^{t, q}(\Omega)$.

\section{A counter-example for generalized Holder-type inequalities in $W^{s, p}$}

Before stating the main theorems, we discuss a simple case which demonstrates that multiplication properties of Sobolev-Slobodeckij spaces can be quite counterintuitive.

Notation: Let $A_{i}$ and $B_{i}(i=1,2)$ and $C$ be Sobolev spaces.

- By writing $A_{1} \times A_{2} \hookrightarrow B_{1} \times B_{2}$ we merely mean that $A_{1} \times A_{2} \subseteq B_{1} \times B_{2}$ and if $u \in A_{1}$ and $v \in A_{2}$, then $\|u\|_{B_{1}}\|v\|_{B_{2}} \preceq\|u\|_{A_{1}}\|v\|_{A_{2}} . \quad\left(A_{1} \times A_{2}=\left\{a_{1} a_{2}: a_{1} \in A_{1}, a_{2} \in\right.\right.$ $\left.\left.A_{2}\right\}\right)$

- By writing $B_{1} \times B_{2} \hookrightarrow C$ we mean that $B_{1} \times B_{2} \subseteq C$ and if $u \in B_{1}$ and $v \in B_{2}$, then $\|u v\|_{C} \preceq\|u\|_{B_{1}}\|v\|_{B_{2}}$.

Theorem 4.1. Suppose $k \in \mathbb{N}_{0}$, and $\frac{1}{p_{1}}+\frac{1}{p_{2}}=\frac{1}{p}$. Then

$$
W^{k, p_{1}}\left(\mathbb{R}^{n}\right) \times W^{k, p_{2}}\left(\mathbb{R}^{n}\right) \longleftrightarrow W^{k, p}\left(\mathbb{R}^{n}\right) .
$$

More generally, if $s \geq 0$, then

$$
H^{s, p_{1}}\left(\mathbb{R}^{n}\right) \times H^{s, p_{2}}\left(\mathbb{R}^{n}\right) \longleftrightarrow H^{s, p}\left(\mathbb{R}^{n}\right) .
$$


Proof of Theorem 4.1. For $k \in \mathbb{N}_{0}$ the claim is a direct consequence of the definition of Sobolev norm, Leibniz formula $\left(\partial^{\alpha}(u v)=\sum_{\beta \leq \alpha}\left(\begin{array}{l}\alpha \\ \beta\end{array}\right) \partial^{\alpha-\beta} u \partial^{\beta} v\right)$, and the Holder's inequality for Lebesgue spaces.

If $s \notin \mathbb{N}_{0}$, then let $k=\lfloor s\rfloor$ and $\theta=s-k$. We have

$$
\begin{aligned}
& H^{k, p_{1}}\left(\mathbb{R}^{n}\right) \times H^{k, p_{2}}\left(\mathbb{R}^{n}\right) \longleftrightarrow H^{k, p}\left(\mathbb{R}^{n}\right), \\
& H^{k+1, p_{1}}\left(\mathbb{R}^{n}\right) \times H^{k+1, p_{2}}\left(\mathbb{R}^{n}\right) \longleftrightarrow H^{k+1, p}\left(\mathbb{R}^{n}\right) .
\end{aligned}
$$

Since

$$
H^{s, p}=\left[H^{k, p}, H^{k+1, p}\right]_{\theta}, \quad H^{s, p_{1}}=\left[H^{k, p_{1}}, H^{k+1, p_{1}}\right]_{\theta}, \quad H^{s, p_{2}}=\left[H^{k, p_{2}}, H^{k+1, p_{2}}\right]_{\theta}
$$

the claim follows from complex interpolation.

Now, we ask the following question: does the claim of Theorem 4.1 hold true for Sobolev-Slobodeckij spaces? More specifically, suppose $s>0, s \notin \mathbb{Z}$, and $\frac{1}{p_{1}}+\frac{1}{p_{2}}=\frac{1}{p}$. Can we conclude that $W^{s, p_{1}}\left(\mathbb{R}^{n}\right) \times W^{s, p_{2}}\left(\mathbb{R}^{n}\right) \hookrightarrow W^{s, p}\left(\mathbb{R}^{n}\right)$ ? Surprisingly, the answer is NO! In what follows we will specialize the argument given in [9] for TriebelLizorkin spaces to the case of Sobolev-Slobodeckij spaces to show that if $s \notin \mathbb{Z}$ (and of course $s>0)$ for $W^{s, p_{1}}\left(\mathbb{R}^{n}\right) \times W^{s_{2}, p_{2}}\left(\mathbb{R}^{n}\right) \hookrightarrow W^{s, p}\left(\mathbb{R}^{n}\right)$ to be true it is necessary to have $p_{1} \leq p$.

Lemma 4.2. Suppose $s>0$ is given. Let $f \in S\left(\mathbb{R}^{n}\right)$ be a function such that

$$
\operatorname{supp} \mathcal{F} f \subseteq\{\xi:|\xi|<\varepsilon\}, \quad f \not \equiv 0 .
$$

If $\varepsilon$ is sufficiently small, then there exists a sequence of functions $\left\{g_{N}\right\}_{N=1}^{\infty}$ (each $g_{N}$ depends on $s$ ) such that for any $p, q>1$

$$
\left\|g_{N}\right\|_{F_{p, q}^{s}}=N^{\frac{1}{q}}\|f\|_{p} \quad \text { and } \quad\left\|g_{N} f\right\|_{F_{p, q}^{s}}=N^{\frac{1}{q}}\left\|f^{2}\right\|_{p}
$$

The construction of $g_{N}$ 's is based on the Littlewood-Paley characterization of Triebel-Lizorkin spaces and can be found in [9].

Proposition 4.3. Suppose $s, s_{2} \geq 0, s \notin \mathbb{Z}$ and $p_{1}, p_{2}, p>1$. If $W^{s, p_{1}}\left(\mathbb{R}^{n}\right) \times$ $W^{s_{2}, p_{2}}\left(\mathbb{R}^{n}\right) \hookrightarrow W^{s, p}\left(\mathbb{R}^{n}\right)$, then $p_{1} \leq p$.

Proof of Proposition 4.3. Note that, since $s \notin \mathbb{Z}$, we have $W^{s, p}=F_{p, p}^{s}$. Consider the product of $f$ and $g_{N}$; by assumption we must have

$$
\left\|g_{N} \cdot f\right\|_{W^{s, p}} \preceq\left\|g_{N}\right\|_{W^{s, p_{1}}}\|f\|_{W^{s_{2}, p_{2}}}
$$

where the implicit constant is independent of $N$. Therefore

$$
N^{\frac{1}{p}}\left\|f^{2}\right\|_{p} \preceq N^{\frac{1}{p_{1}}}\|f\|_{p_{1}}\|f\|_{W^{s_{2}, p_{2}}} .
$$


So for all $N \in \mathbb{N}$

$$
0<\frac{\left\|f^{2}\right\|_{p}}{\|f\|_{p_{1}}\|f\|_{W^{s_{2}, p_{2}}}} \preceq N^{\frac{1}{p_{1}}-\frac{1}{p}},
$$

which implies that $p_{1} \leq p$.

Remark 4.4. Proposition 4.3, in part, shows that the claim of Theorem 1.4.4.2 of [7] (in the generality that is stated in [7]) does not hold true. Also the claim stated in part (d) of page 47 in [2] (in the generality that is stated in [2]) does not hold true.

\section{Sufficient conditions for $\boldsymbol{H}^{s_{1}, p_{1}} \times H^{s_{2}, p_{2}} \hookrightarrow H^{s, p}, s \geq 0, s \in \mathbb{R}$}

We start our main theorems by a theorem on multiplication in spaces $H^{s, p}\left(\mathbb{R}^{n}\right)$ with $s \geq 0$. The reason that we begin with a theorem on Bessel potential spaces is that although for these spaces the situation is considerably simpler (comparing to Sobolev-Slobodeckij spaces), it showcases the main ideas without encountering technical difficulties. The aforementioned simplicity is due to the fact that we have a uniform formula for the space $\left[H^{s_{0}, p_{0}}, H^{s_{1}, p_{1}}\right]_{\theta}$ regardless of whether each of $s_{0}$, $s_{1}$, or $(1-\theta) s_{0}+\theta s_{1}$ is an integer or not. This first result is classical and wellknown; however, the following fairly short proof based on complex interpolation and embedding theorems does not appear to be in the literature, so we include it.

Theorem 5.1. (Pointwise multiplication in spaces $H^{s, p}\left(\mathbb{R}^{n}\right)$ with $s \geq 0$ ) Assume $s_{i}, s$ and $1<p_{i} \leq p<\infty(i=1,2)$ are real numbers satisfying

(i) $s_{i} \geq s$,

(ii) $s \geq 0$,

(iii) $s_{i}-s \geq n\left(\frac{1}{p_{i}}-\frac{1}{p}\right)$,

(iv) $s_{1}+s_{2}-s>n\left(\frac{1}{p_{1}}+\frac{1}{p_{2}}-\frac{1}{p}\right)$.

Then, if $u \in H^{s_{1}, p_{1}}\left(\mathbb{R}^{n}\right)$ and $v \in H^{s_{2}, p_{2}}\left(\mathbb{R}^{n}\right), u v \in H^{s, p}\left(\mathbb{R}^{n}\right)$, and moreover, the pointwise multiplication of functions is a continuous bilinear map

$$
H^{s_{1}, p_{1}}\left(\mathbb{R}^{n}\right) \times H^{s_{2}, p_{2}}\left(\mathbb{R}^{n}\right) \longrightarrow H^{s, p}\left(\mathbb{R}^{n}\right) .
$$

Proof of Theorem 5.1. Our proof consists of two steps. In the first step we consider the special case $p_{1}=p_{2}=p$, and then in the second step we prove the general case based on the special case that is proved in Step 1.

Step 1: Here we want to prove the theorem for the special case $p=p_{1}=p_{2}$. In this case the assumptions can be rewritten as follows:

$$
s_{1}, s_{2} \geq s \geq 0, \quad s_{1}+s_{2}-s>\frac{n}{p} .
$$

In order to proceed, we state and prove a simple lemma. 


\section{Lemma 5.2.}

$$
\begin{aligned}
& \forall \varepsilon>0 \forall t \in\left[0, \frac{n}{p}\right] \quad H^{t, p}\left(\mathbb{R}^{n}\right) \times H^{\frac{n}{p}+\varepsilon, p}\left(\mathbb{R}^{n}\right) \longleftrightarrow H^{t, p}\left(\mathbb{R}^{n}\right) . \\
& \forall \varepsilon>0 \forall t \in\left[0, \frac{n}{p}\right] \quad H^{\frac{n}{p}+\varepsilon, p}\left(\mathbb{R}^{n}\right) \times H^{t, p}\left(\mathbb{R}^{n}\right) \longleftrightarrow H^{t, p}\left(\mathbb{R}^{n}\right) .
\end{aligned}
$$

Proof of the Lemma 5.2. Clearly it is enough to prove the first statement. Let $\varepsilon>0$ be given. Since $\frac{n}{p}+\varepsilon>\frac{n}{p}, H^{\frac{n}{p}+\varepsilon, p}\left(\mathbb{R}^{n}\right)$ is an algebra and

$$
H^{\frac{n}{p}+\varepsilon, p}\left(\mathbb{R}^{n}\right) \times H^{\frac{n}{p}+\varepsilon, p}\left(\mathbb{R}^{n}\right) \longleftrightarrow H^{\frac{n}{p}+\varepsilon, p}\left(\mathbb{R}^{n}\right) .
$$

Also $H^{\frac{n}{p}+\varepsilon, p}\left(\mathbb{R}^{n}\right) \hookrightarrow L^{\infty}\left(\mathbb{R}^{n}\right)$. Hence

$$
H^{\frac{n}{p}+\varepsilon, p}\left(\mathbb{R}^{n}\right) \times H^{0, p}\left(\mathbb{R}^{n}\right) \longleftrightarrow H^{0, p}\left(\mathbb{R}^{n}\right) \quad\left(L^{\infty} \times L^{p} \longleftrightarrow L^{p}\right) .
$$

By complex interpolation between (5.1) and (5.2) we get

$$
\forall \theta \in[0,1] \quad H^{\frac{n}{p}+\varepsilon, p}\left(\mathbb{R}^{n}\right) \times H^{\theta\left(\frac{n}{p}+\varepsilon\right), p}\left(\mathbb{R}^{n}\right) \longleftrightarrow H^{\theta\left(\frac{n}{p}+\varepsilon\right), p}\left(\mathbb{R}^{n}\right)
$$

which clearly implies the claim of the Lemma 5.2.

Now, using the above lemma, we can prove the theorem for the special case $p=p_{1}=p_{2}$. To this end we consider two cases:

Case $1 s>\frac{n}{p}$ : If $s>\frac{n}{p}$, then $H^{s, p}\left(\mathbb{R}^{n}\right)$ is an algebra and we can write

$$
\begin{aligned}
H^{s_{1}, p_{1}}\left(\mathbb{R}^{n}\right) \times H^{s_{2}, p_{2}}\left(\mathbb{R}^{n}\right) & \left.\longleftrightarrow H^{s, p}\left(\mathbb{R}^{n}\right) \times H^{s, p}\left(\mathbb{R}^{n}\right) \quad \text { (by assumption } s_{1}, s_{2} \geq s\right) \\
& \longleftrightarrow H^{s, p}\left(\mathbb{R}^{n}\right) .
\end{aligned}
$$

Case $2 s \leq \frac{n}{p}$ : Let $\varepsilon=s_{1}+s_{2}-s-\frac{n}{p}>0$. By Lemma 5.2 we have

$$
\begin{aligned}
& H^{s, p}\left(\mathbb{R}^{n}\right) \times H^{\frac{n}{p}+\varepsilon, p}\left(\mathbb{R}^{n}\right) \longleftrightarrow H^{s, p}\left(\mathbb{R}^{n}\right) . \\
& H^{\frac{n}{p}+\varepsilon, p}\left(\mathbb{R}^{n}\right) \times H^{s, p}\left(\mathbb{R}^{n}\right) \longleftrightarrow H^{s, p}\left(\mathbb{R}^{n}\right) .
\end{aligned}
$$

Note that

$$
s \leq s_{2} \Longrightarrow s_{1} \leq s_{1}+s_{2}-s \Longrightarrow s_{1} \leq \frac{n}{p}+\varepsilon .
$$

So, there exists $\theta \in[0,1]$ such that $(1-\theta) s+\theta\left(\frac{n}{p}+\varepsilon\right)=s_{1}$. Clearly

$$
\left[(1-\theta) s+\theta\left(\frac{n}{p}+\varepsilon\right)\right]+\left[(1-\theta)\left(\frac{n}{p}+\varepsilon\right)+\theta s\right]=s+\frac{n}{p}+\varepsilon=s_{1}+s_{2} .
$$

That is, $s_{1}+\left[(1-\theta)\left(\frac{n}{p}+\varepsilon\right)+\theta s\right]=s_{1}+s_{2}$ which means that $(1-\theta)\left(\frac{n}{p}+\varepsilon\right)+\theta s=$ $s_{2}$. Consequently

$$
\left[H^{s, p}\left(\mathbb{R}^{n}\right), H^{\frac{n}{p}+\varepsilon, p}\left(\mathbb{R}^{n}\right)\right]_{\theta}=H^{s_{1}, p}\left(\mathbb{R}^{n}\right)
$$




$$
\left[H^{\frac{n}{p}+\varepsilon, p}\left(\mathbb{R}^{n}\right), H^{s, p}\left(\mathbb{R}^{n}\right)\right]_{\theta}=H^{s_{2}, p}\left(\mathbb{R}^{n}\right) .
$$

So, using complex interpolation, (5.3), and (5.4) we get

$$
H^{s_{1}, p_{1}}\left(\mathbb{R}^{n}\right) \times H^{s_{2}, p_{2}}\left(\mathbb{R}^{n}\right) \longleftrightarrow H^{s, p}\left(\mathbb{R}^{n}\right) .
$$

Step 2: Now we are in the position to prove the general case. Let

$$
\tilde{s}_{1}=s_{1}-\frac{n}{p_{1}}+\frac{n}{p}, \quad \tilde{s}_{2}=s_{2}-\frac{n}{p_{2}}+\frac{n}{p} .
$$

We just need to prove the following claim:

\section{Claim.}

(i) $H^{\tilde{s}_{1}, p}\left(\mathbb{R}^{n}\right) \times H^{\tilde{s}_{2}, p}\left(\mathbb{R}^{n}\right) \hookrightarrow H^{s, p}\left(\mathbb{R}^{n}\right)$.

(ii) $H^{s_{1}, p_{1}}\left(\mathbb{R}^{n}\right) \hookrightarrow H^{\tilde{s}_{1}, p}\left(\mathbb{R}^{n}\right)$.

(iii) $H^{s_{2}, p_{2}}\left(\mathbb{R}^{n}\right) \hookrightarrow H^{\tilde{s}_{2}, p}\left(\mathbb{R}^{n}\right)$.

Indeed, if we prove the above claim, then

$$
H^{s_{1}, p_{1}}\left(\mathbb{R}^{n}\right) \times H^{s_{2}, p_{2}}\left(\mathbb{R}^{n}\right) \longleftrightarrow H^{\tilde{s}_{1}, p}\left(\mathbb{R}^{n}\right) \times H^{\tilde{s}_{2}, p}\left(\mathbb{R}^{n}\right) \longleftrightarrow H^{s, p}\left(\mathbb{R}^{n}\right) .
$$

Proof of $(i)$. By Step 1 we need to check the following items:

$$
\begin{aligned}
& \tilde{s}_{1} \geq s \quad\left(\text { true because } s_{1}-s \geq n\left(\frac{1}{p_{1}}-\frac{1}{p}\right)\right) \\
& \tilde{s}_{2} \geq s \quad\left(\text { true because } s_{2}-s \geq n\left(\frac{1}{p_{2}}-\frac{1}{p}\right)\right) \\
& \tilde{s}_{1}+\tilde{s}_{2}-s>\frac{n}{p}
\end{aligned}
$$

The last item is true because

$$
\begin{aligned}
s_{1}+s_{2}-s>n\left(\frac{1}{p_{1}}+\frac{1}{p_{2}}-\frac{1}{p}\right) & \Longrightarrow\left(s_{1}-\frac{n}{p_{1}}+\frac{n}{p}\right)+\left(s_{2}-\frac{n}{p_{2}}+\frac{n}{p}\right)-s>\frac{n}{p} \\
& \Longrightarrow \tilde{s}_{1}+\tilde{s}_{2}-s>\frac{n}{p} . \square
\end{aligned}
$$

Proof of (ii). According to Embedding Theorem I we must check the following items:

$$
\begin{aligned}
& p_{1} \leq p \quad(\text { true by assumption }) \\
& s_{1} \geq \tilde{s}_{1} \quad\left(\text { true because } p \geq p_{1} \Longrightarrow \frac{n}{p_{1}} \geq \frac{n}{p} \Longrightarrow s_{1} \geq s_{1}-\frac{n}{p_{1}}+\frac{n}{p}\right) \\
& s_{1}-\frac{n}{p_{1}} \geq \tilde{s}_{1}-\frac{n}{p} \quad\left(\text { true because } \tilde{s}_{1}-\frac{n}{p}=s_{1}-\frac{n}{p_{1}}+\frac{n}{p}-\frac{n}{p}=s_{1}-\frac{n}{p_{1}}\right)
\end{aligned}
$$

Proof of (iii). Completely analogous to the proof of the previous item! This completes the proof of the Claim. 


\section{Sufficient conditions for $W^{s_{1}, p_{1}} \times W^{s_{2}, p_{2}} \hookrightarrow W^{s, p}, s \geq 0, s \in \mathbb{N}_{0}$}

We now consider the case where the product belongs to a Sobolev space with integer smoothness index. The proof of the following theorem is based on the classical definition of Sobolev spaces, Holder's inequality for Lebesgue spaces, and previously stated embedding theorems.

Theorem 6.1. Let $s_{i}, s$ and $1 \leq p, p_{i}<\infty(i=1,2)$ be real numbers satisfying

(i) $s_{i} \geq s \geq 0$

(ii) $s \in \mathbb{N}_{0}$,

(iii) $s_{i}-s \geq n\left(\frac{1}{p_{i}}-\frac{1}{p}\right)$,

(iv) $s_{1}+s_{2}-s>n\left(\frac{1}{p_{1}}+\frac{1}{p_{2}}-\frac{1}{p}\right) \geq 0$.

where the strictness of the inequalities in items (iii) and (iv) can be interchanged.

Then, if $u \in W^{s_{1}, p_{1}}\left(\mathbb{R}^{n}\right)$ and $v \in W^{s_{2}, p_{2}}\left(\mathbb{R}^{n}\right), u v \in W^{s, p}\left(\mathbb{R}^{n}\right)$ and moreover the pointwise multiplication of functions is a continuous bilinear map

$$
W^{s_{1}, p_{1}}\left(\mathbb{R}^{n}\right) \times W^{s_{2}, p_{2}}\left(\mathbb{R}^{n}\right) \longrightarrow W^{s, p}\left(\mathbb{R}^{n}\right) .
$$

Remark 6.2. Note that $p_{i}$ is not required to be less than or equal to $p$ in the statement of Theorem 6.1. It is the restriction that $s$ be an integer in the theorem that makes it possible to remove the ordering between $p_{i}$ and $p$. We will see below in Theorem 7.4 that alternatively, one can restrict consideration to a bounded domain $\Omega$ in place of $\mathbb{R}^{n}$, allowing $s$ to be noninteger, yet still removing the ordering restriction between $p_{i}$ and $p$.

Proof of Theorem 6.1. Let $u \in W^{s_{1}, p_{1}}\left(\mathbb{R}^{n}\right)$ and $v \in W^{s_{2}, p_{2}}\left(\mathbb{R}^{n}\right)$. Our goal is to prove that $\|u v\|_{s, p} \preceq\|u\|_{s_{1}, p_{1}}\|v\|_{s_{2}, p_{2}}$. We have

$$
\|u v\|_{s, p}=\sum_{|\alpha| \leq s}\left\|\partial^{\alpha}(u v)\right\|_{p} .
$$

So, it is enough to prove that for all $|\alpha| \leq s,\left\|\partial^{\alpha}(u v)\right\|_{p} \preceq\|u\|_{s_{1}, p_{1}}\|v\|_{s_{2}, p_{2}}$. For now let's assume $v \in C_{c}^{\infty}\left(\mathbb{R}^{n}\right)$. So we can use the Leibniz formula (see e.g. [1]) to write

$$
\partial^{\alpha}(u v)=\sum_{\beta \leq \alpha}\left(\begin{array}{l}
\alpha \\
\beta
\end{array}\right) \partial^{\alpha-\beta} u \partial^{\beta} v .
$$

Thus we just need to show that

$$
\forall|\alpha| \leq s \forall \beta \leq \alpha \quad\left\|\partial^{\alpha-\beta} u \partial^{\beta} v\right\|_{p} \preceq\|u\|_{s_{1}, p_{1}}\|v\|_{s_{2}, p_{2}} .
$$

Fix $\alpha, \beta \in \mathbb{N}_{0}^{n}$ such that $|\alpha| \leq s$ and $\beta \leq \alpha$. In what follows we will prove the following claim: 
Claim. There exist $r \in[1, \infty]$ and $q \in[1, \infty]$ such that

$$
\frac{1}{r}+\frac{1}{q}=\frac{1}{p}, \quad W^{s_{1}-|\alpha-\beta|, p_{1}}\left(\mathbb{R}^{n}\right) \longleftrightarrow L^{r}\left(\mathbb{R}^{n}\right), W^{s_{2}-|\beta|, p_{2}}\left(\mathbb{R}^{n}\right) \longleftrightarrow L^{q}\left(\mathbb{R}^{n}\right) .
$$

For the moment, let's assume the above claim is true. Then

$$
\begin{aligned}
& u \in W^{s_{1}, p_{1}}\left(\mathbb{R}^{n}\right) \Longrightarrow \partial^{\alpha-\beta} u \in W^{s_{1}-|\alpha-\beta|, p_{1}}\left(\mathbb{R}^{n}\right) \longleftrightarrow L^{r}\left(\mathbb{R}^{n}\right), \\
& v \in W^{s_{2}, p_{2}}\left(\mathbb{R}^{n}\right) \Longrightarrow \partial^{\beta} v \in W^{s_{2}-|\beta|, p_{2}}\left(\mathbb{R}^{n}\right) \longleftrightarrow L^{q}\left(\mathbb{R}^{n}\right),
\end{aligned}
$$

and therefore

$$
\begin{aligned}
\left\|\partial^{\alpha-\beta} u \partial^{\beta} v\right\|_{p} \leq\left\|\partial^{\alpha-\beta} u\right\|_{r}\left\|\partial^{\beta} v\right\|_{q} & \preceq\left\|\partial^{\alpha-\beta} u\right\|_{s_{1}-|\alpha-\beta|, p_{1}}\left\|\partial^{\beta} v\right\|_{s_{2}-|\beta|, p_{2}} \\
& \preceq\|u\|_{s_{1}, p_{1}}\|v\|_{s_{2}, p_{2}} .
\end{aligned}
$$

So, it is enough to prove the above claim. We consider two cases separately:

Case 1: $s_{i}-s>n\left(\frac{1}{p_{i}}-\frac{1}{p}\right)(i=1,2)$ and $s_{1}+s_{2}-s \geq n\left(\frac{1}{p_{1}}+\frac{1}{p_{2}}-\frac{1}{p}\right) \geq 0$.

As a direct consequence of assumptions we have

$$
\begin{aligned}
& \frac{1}{p_{1}}-\frac{s_{1}-|\alpha-\beta|}{n} \leq \frac{1}{p_{1}}-\frac{s_{1}-s}{n}<\frac{1}{p} . \\
& \frac{1}{p}-\frac{1}{p_{2}}+\frac{s_{2}-|\beta|}{n} \geq \frac{1}{p}-\frac{1}{p_{2}}+\frac{s_{2}-s}{n}>0 .
\end{aligned}
$$

In what follows we will show that there exist $r \in[1, \infty)$ and $q \in[1, \infty)$ that satisfy (6.1). According to Theorem 3.8 it is enough to show that there exist $r$ and $q$ that satisfy the following conditions:

$$
\begin{aligned}
& 0<\frac{1}{r} \leq 1, \quad 0<\frac{1}{q} \leq 1, \quad \frac{1}{r}+\frac{1}{q}=\frac{1}{p} \\
& \frac{1}{r} \leq \frac{1}{p_{1}}, \quad \frac{1}{q} \leq \frac{1}{p_{2}}, \\
& s_{1}-|\alpha-\beta|-\frac{n}{p_{1}} \geq 0-\frac{n}{r}, \quad s_{2}-|\beta|-\frac{n}{p_{2}} \geq 0-\frac{n}{q} .
\end{aligned}
$$

In fact, if we let $R=\frac{1}{r}$ and $Q=\frac{1}{q}$, then our goal is to show that there exist $0<R \leq 1$ and $0<Q \leq 1$ such that

$$
R+Q=\frac{1}{p}, \quad \frac{1}{p_{1}}-\frac{s_{1}-|\alpha-\beta|}{n} \leq R \leq \frac{1}{p_{1}}, \quad \frac{1}{p_{2}}-\frac{s_{2}-|\beta|}{n} \leq Q \leq \frac{1}{p_{2}} .
$$

Note that since $\frac{1}{p_{1}} \leq 1$ and $\frac{1}{p_{2}} \leq 1$, conditions $R \leq 1$ and $Q \leq 1$ are superfluous. So, we need to show that there exists $0<R<\frac{1}{p}$ such that

$$
\frac{1}{p_{1}}-\frac{s_{1}-|\alpha-\beta|}{n} \leq R \leq \frac{1}{p_{1}}
$$




$$
\frac{1}{p_{2}}-\frac{s_{2}-|\beta|}{n} \leq \frac{1}{p}-R \leq \frac{1}{p_{2}} \quad\left(\Longleftrightarrow \frac{1}{p}-\frac{1}{p_{2}} \leq R \leq \frac{1}{p}-\frac{1}{p_{2}}+\frac{s_{2}-|\beta|}{n}\right) .
$$

Consequently, it is enough to show that the following intersection is nonempty:

$$
\left(0, \frac{1}{p}\right) \cap\left[\frac{1}{p_{1}}-\frac{s_{1}-|\alpha-\beta|}{n}, \frac{1}{p_{1}}\right] \cap\left[\frac{1}{p}-\frac{1}{p_{2}}, \frac{1}{p}-\frac{1}{p_{2}}+\frac{s_{2}-|\beta|}{n}\right] .
$$

Note that by (6.2), $\frac{1}{p_{1}}-\frac{s_{1}-|\alpha-\beta|}{n}<\frac{1}{p}$ and so the first intersection is nonempty. We may consider four cases:

(i) $\frac{1}{p_{1}}-\frac{s_{1}-|\alpha-\beta|}{n} \leq 0, \frac{1}{p_{1}}<\frac{1}{p}$ :

$$
\left(0, \frac{1}{p}\right) \cap\left[\frac{1}{p_{1}}-\frac{s_{1}-|\alpha-\beta|}{n}, \frac{1}{p_{1}}\right]=\left(0, \frac{1}{p_{1}}\right] .
$$

Now note that by assumption $\frac{1}{p_{1}} \geq \frac{1}{p}-\frac{1}{p_{2}}$ and also by (6.3), $\frac{1}{p}-\frac{1}{p_{2}}+\frac{s_{2}-|\beta|}{n}>0$. Hence

$$
\left(0, \frac{1}{p_{1}}\right] \cap\left[\frac{1}{p}-\frac{1}{p_{2}}, \frac{1}{p}-\frac{1}{p_{2}}+\frac{s_{2}-|\beta|}{n}\right] \neq \varnothing .
$$

(ii) $\frac{1}{p_{1}}-\frac{s_{1}-|\alpha-\beta|}{n} \leq 0, \frac{1}{p_{1}} \geq \frac{1}{p}$ :

$$
\left(0, \frac{1}{p}\right) \cap\left[\frac{1}{p_{1}}-\frac{s_{1}-|\alpha-\beta|}{n}, \frac{1}{p_{1}}\right]=\left(0, \frac{1}{p}\right) .
$$

Clearly $\frac{1}{p}>\frac{1}{p}-\frac{1}{p_{2}}$ and also by (6.3), $\frac{1}{p}-\frac{1}{p_{2}}+\frac{s_{2}-|\beta|}{n}>0$. Hence

$$
\left(0, \frac{1}{p}\right) \cap\left[\frac{1}{p}-\frac{1}{p_{2}}, \frac{1}{p}-\frac{1}{p_{2}}+\frac{s_{2}-|\beta|}{n}\right] \neq \varnothing .
$$

(iii) $\frac{1}{p_{1}}-\frac{s_{1}-|\alpha-\beta|}{n}>0, \frac{1}{p} \leq \frac{1}{p_{1}}$ :

$$
\left(0, \frac{1}{p}\right) \cap\left[\frac{1}{p_{1}}-\frac{s_{1}-|\alpha-\beta|}{n}, \frac{1}{p_{1}}\right]=\left[\frac{1}{p_{1}}-\frac{s_{1}-|\alpha-\beta|}{n}, \frac{1}{p}\right) .
$$

Clearly $\frac{1}{p}>\frac{1}{p}-\frac{1}{p_{2}}$ and also by assumption $s_{1}+s_{2}-s \geq n\left(\frac{1}{p_{1}}+\frac{1}{p_{2}}-\frac{1}{p}\right)$ and so $\frac{1}{p_{1}}-$ $\frac{s_{1}-|\alpha-\beta|}{n} \leq \frac{1}{p}-\frac{1}{p_{2}}+\frac{s_{2}-|\beta|}{n}$. Consequently,

$$
\left[\frac{1}{p_{1}}-\frac{s_{1}-|\alpha-\beta|}{n}, \frac{1}{p}\right) \cap\left[\frac{1}{p}-\frac{1}{p_{2}}, \frac{1}{p}-\frac{1}{p_{2}}+\frac{s_{2}-|\beta|}{n}\right] \neq \varnothing .
$$


(iv) $\frac{1}{p_{1}}-\frac{s_{1}-|\alpha-\beta|}{n}>0, \frac{1}{p_{1}}<\frac{1}{p}$ :

$$
\left(0, \frac{1}{p}\right) \cap\left[\frac{1}{p_{1}}-\frac{s_{1}-|\alpha-\beta|}{n}, \frac{1}{p_{1}}\right]=\left[\frac{1}{p_{1}}-\frac{s_{1}-|\alpha-\beta|}{n}, \frac{1}{p_{1}}\right] .
$$

By assumption $\frac{1}{p_{1}} \geq \frac{1}{p}-\frac{1}{p_{2}}$ and also (exactly the same as the previous item) $\frac{1}{p_{1}}-$ $\frac{s_{1}-|\alpha-\beta|}{n} \leq \frac{1}{p}-\frac{1}{p_{2}}+\frac{s_{2}-|\beta|}{n}$. Consequently,

$$
\left[\frac{1}{p_{1}}-\frac{s_{1}-|\alpha-\beta|}{n}, \frac{1}{p_{1}}\right] \cap\left[\frac{1}{p}-\frac{1}{p_{2}}, \frac{1}{p}-\frac{1}{p_{2}}+\frac{s_{2}-|\beta|}{n}\right] \neq \varnothing .
$$

Case 2: $s_{i}-s \geq n\left(\frac{1}{p_{i}}-\frac{1}{p}\right)(i=1,2)$ and $s_{1}+s_{2}-s>n\left(\frac{1}{p_{1}}+\frac{1}{p_{2}}-\frac{1}{p}\right) \geq 0$.

If $s_{i}-s>n\left(\frac{1}{p_{i}}-\frac{1}{p}\right)(i=1,2)$, then the proof of previous case works. So, we just need to consider the following cases:

(i) $s_{1}-s=n\left(\frac{1}{p_{1}}-\frac{1}{p}\right), s_{2}-s \neq n\left(\frac{1}{p_{2}}-\frac{1}{p}\right):$ If $|\alpha-\beta|<s$, then the proof of Case 1 works. In fact, note that the proof of Case 1 was based on the inequalities $\frac{1}{p_{1}}-\frac{s_{1}-|\alpha-\beta|}{n}<\frac{1}{p}$ and $\frac{1}{p}-\frac{1}{p_{2}}+\frac{s_{2}-|\beta|}{n}>0((6.2)$ and (6.3)) and both inequalities hold true in this case: the second inequality is true because as in Case $1 s_{2}-s>n\left(\frac{1}{p_{2}}-\frac{1}{p}\right)$, and the first inequality is true because

$$
\frac{1}{p_{1}}-\frac{s_{1}-|\alpha-\beta|}{n}<\frac{1}{p_{1}}-\frac{s_{1}-s}{n} \leq \frac{1}{p} .
$$

So, we may assume $|\alpha-\beta|=s$. Since $|\alpha| \leq s$ and $\beta \leq \alpha$, this is possible only if $|\alpha|=s$ and $|\beta|=0$.

By assumption $s_{1}+s_{2}-s>n\left(\frac{1}{p_{1}}+\frac{1}{p_{2}}-\frac{1}{p}\right)$, so $s_{2}>\frac{n}{p_{2}}$. Also $s_{1}-s \geq 0$ and therefore $p_{1} \leq p$. Consequently

$$
W^{s_{1}-s, p_{1}}\left(\mathbb{R}^{n}\right) \longleftrightarrow L^{p}\left(\mathbb{R}^{n}\right), \quad W^{s_{2}, p_{2}}\left(\mathbb{R}^{n}\right) \longleftrightarrow L^{\infty}\left(\mathbb{R}^{n}\right) .
$$

That is, (6.1) is satisfied with $r=p$ and $q=\infty$. (Note that $|\alpha-\beta|=s$ and $|\beta|=0$.)

(ii) $s_{2}-s=n\left(\frac{1}{p_{2}}-\frac{1}{p}\right), s_{1}-s \neq n\left(\frac{1}{p_{1}}-\frac{1}{p}\right)$ : If $|\beta|<s$, then the proof of Case 1 works (again because inequalities $\frac{1}{p_{1}}-\frac{s_{1}-|\alpha-\beta|}{n}<\frac{1}{p}$ and $\frac{1}{p}-\frac{1}{p_{2}}+\frac{s_{2}-|\beta|}{n}>0$ hold true). So, we may assume $|\beta|=s$. Since $|\alpha| \leq s$ and $\beta \leq \alpha$, this is possible only if $|\alpha|=s$ and $\beta=\alpha$. Exactly similar to [(i)], one can show that $r=\infty$ and $q=p$ satisfy (6.1).

(iii) $s_{1}-s=n\left(\frac{1}{p_{1}}-\frac{1}{p}\right), s_{2}-s=n\left(\frac{1}{p_{2}}-\frac{1}{p}\right):$ If $|\alpha-\beta|<s,|\beta|<s$, then the proof of Case 1 works. If $|\alpha-\beta|=s$ and $|\beta|<s$, then the argument given in item [(i)] works. If $|\alpha-\beta|<s$ and $|\beta|=s$, then the argument given in item [(ii)] works. Also note that, since $|\alpha| \leq s$ and $\beta \leq \alpha$, it is not possible to have $|\alpha-\beta|=|\beta|=s$. 
So, we proved $\|u v\|_{s, p} \preceq\|u\|_{s_{1}, p_{1}}\|v\|_{s_{2}, p_{2}}$ for $v \in C_{c}^{\infty}\left(\mathbb{R}^{n}\right)$ and $u \in W^{s_{1}, p_{1}}\left(\mathbb{R}^{n}\right)$. Now, suppose $v$ is an arbitrary element of $W^{s_{2}, p_{2}}\left(\mathbb{R}^{n}\right)$. There exists a sequence $v_{j} \in$ $C_{c}^{\infty}\left(\mathbb{R}^{n}\right)$ such that $v_{j} \rightarrow v$ in $W^{s_{2}, p_{2}}\left(\mathbb{R}^{n}\right)$. We have

$$
\left\|u v_{j}-u v_{j^{\prime}}\right\|_{s, p} \preceq\left\|v_{j}-v_{j^{\prime}}\right\|_{s_{2}, p_{2}}\|u\|_{s_{1}, p_{1}} .
$$

Therefore, $u v_{j}$ is a Cauchy sequence in $W^{s, p}\left(\mathbb{R}^{n}\right)$ and so $u v_{j}$ converges to an element $w \in W^{s, p}\left(\mathbb{R}^{n}\right)$. Since $W^{s, p}\left(\mathbb{R}^{n}\right) \hookrightarrow L^{p}\left(\mathbb{R}^{n}\right), u v_{j} \rightarrow w$ in $L^{p}\left(\mathbb{R}^{n}\right)$. Hence, there exists a subsequence $u \tilde{v}_{j}$ that converges to $w$ almost everywhere. On the other hand,

$$
\begin{aligned}
\tilde{v}_{j} \longrightarrow v \text { in } W^{s_{2}, p_{2}}\left(\mathbb{R}^{n}\right) & \longrightarrow \tilde{v}_{j} \longrightarrow v \text { in } L^{p_{2}}\left(\mathbb{R}^{n}\right) \\
& \Longrightarrow \exists \text { a subsequence } \tilde{\tilde{v}}_{j} \text { such that } \tilde{\tilde{v}}_{j} \longrightarrow v \text { a.e. }
\end{aligned}
$$

Consequently $u \tilde{\tilde{v}}_{j} \rightarrow u v$ a.e. and $u \tilde{\tilde{v}}_{j} \rightarrow w$ a.e., and so $u v=w$ a.e. as well. Therefore, $u v \in W^{s, p}\left(\mathbb{R}^{n}\right)$ and

$$
\begin{aligned}
&\|u v\|_{s, p}=\left\|\lim _{j \rightarrow \infty}\left(u v_{j}\right)\right\|_{s, p}=\lim _{j \rightarrow \infty}\left\|\left(u v_{j}\right)\right\|_{s, p} \preceq \lim _{j \rightarrow \infty}\left\|v_{j}\right\|_{s_{2}, p_{2}}\|u\|_{s_{1}, p_{1}} \\
&=\|v\|_{s_{2}, p_{2}}\|u\|_{s_{1}, p_{1}} . \quad \square
\end{aligned}
$$

Corollary 6.3. Using extension operators, one can easily show that the above result holds also for Sobolev spaces on any bounded domain with Lipschitz continuous boundary. Indeed, if $P_{1}: W^{s_{1}, p_{1}}(\Omega) \rightarrow W^{s_{1}, p_{1}}\left(\mathbb{R}^{n}\right)$ and $P_{2}: W^{s_{2}, p_{2}}(\Omega) \rightarrow W^{s_{2}, p_{2}}\left(\mathbb{R}^{n}\right)$ are extension operators, then $\left.\left(P_{1} u\right)\left(P_{2} v\right)\right|_{\Omega}=u v$ and therefore

$$
\begin{aligned}
\|u v\|_{W^{s, p}(\Omega)} \leq\left\|\left(P_{1} u\right)\left(P_{2} v\right)\right\|_{W^{s, p}\left(\mathbb{R}^{n}\right)} & \preceq\left\|P_{1} u\right\|_{W^{s_{1}, p_{1}}\left(\mathbb{R}^{n}\right)}\left\|P_{2} v\right\|_{W^{s_{2}, p_{2}}\left(\mathbb{R}^{n}\right)} \\
& \preceq\|u\|_{W^{s_{1}, p_{1}(\Omega)}}\|v\|_{W^{s_{2}, p_{2}}(\Omega)} .
\end{aligned}
$$

\section{Sufficient conditions for $W^{s_{1}, p_{1}} \times W^{s_{2}, p_{2}} \hookrightarrow W^{s, p}, s \geq 0, s \in \mathbb{R}$}

As noted earlier in Remark 6.2 just following Theorem 6.1, on that theorem $p_{i}$ was not required to be less than or equal to $p$. It is the restriction that $s$ be an integer in Theorem 6.1 that makes it possible to remove the ordering between $p_{i}$ and $p$. We see in Theorem 7.4 below that alternatively, one can restrict consideration to a bounded domain $\Omega$ in place of $\mathbb{R}^{n}$, allowing $s$ to be noninteger, yet still removing the ordering restriction between $p_{i}$ and $p$. First we consider the case of unbounded domains and real exponents, with the ordering restriction between $p_{i}$ and $p$. It is worth mentioning that, as opposed to the proofs of the similar results in the literature which are based on Littlewood-Paley theory and Besov spaces, the proofs presented here are based on interpolation theory and embedding theorems without any reference to Littlewood-Paley theory.

Before proceeding any further, first we need to state two lemmas: 
Lemma 7.1. Let $\Omega$ be a bounded open subset of $\mathbb{R}^{n}$ with Lipschitz continuous boundary, or $\Omega=\mathbb{R}^{n}$.

$$
\begin{aligned}
& \forall \varepsilon>0, \forall m \in\left[0, \frac{n}{p}\right] \cap \mathbb{Z}, \quad W^{m, p}(\Omega) \times W^{\frac{n}{p}+\varepsilon, p}(\Omega) \longleftrightarrow W^{m, p}(\Omega) . \\
& \forall \varepsilon>0, \forall m \in\left[0, \frac{n}{p}\right] \cap \mathbb{Z}, \quad W^{\frac{n}{p}+\varepsilon, p}(\Omega) \times W^{m, p}(\Omega) \longleftrightarrow W^{m, p}(\Omega) .
\end{aligned}
$$

Proof of Lemma \%.1. This is a direct consequence of the previous theorem.

Lemma 7.2. Let $\Omega$ be a bounded open subset of $\mathbb{R}^{n}$ with Lipschitz continuous boundary, or $\Omega=\mathbb{R}^{n}$.

$$
\begin{aligned}
& \forall \varepsilon>0, \forall s \in\left[0, \frac{n}{p}\right], \quad W^{s, p}(\Omega) \times W^{\frac{n}{p}+\varepsilon, p}(\Omega) \longleftrightarrow W^{s, p}(\Omega) . \\
& \forall \varepsilon>0, \forall s \in\left[0, \frac{n}{p}\right], \quad W^{\frac{n}{p}+\varepsilon, p}(\Omega) \times W^{s, p}(\Omega) \longleftrightarrow W^{s, p}(\Omega) .
\end{aligned}
$$

Proof of Lemma 7.2. Clearly, we just need to prove the first statement. Let $\varepsilon>0$ and $s \in\left[0, \frac{n}{p}\right]$ be given. By Lemma 7.1 if $s \in \mathbb{Z}$ the claim holds true. So, we may assume $s \notin \mathbb{Z}$. Since $\frac{n}{p}+\varepsilon>\frac{n}{p}, W^{\frac{n}{p}+\varepsilon, p}(\Omega)$ is an algebra and

$$
W^{\frac{n}{p}+\varepsilon, p}(\Omega) \times W^{\frac{n}{p}+\varepsilon, p}(\Omega) \longleftrightarrow W^{\frac{n}{p}+\varepsilon, p}(\Omega) .
$$

Also $W^{\frac{n}{p}+\varepsilon, p}(\Omega) \hookrightarrow L^{\infty}(\Omega)$. Hence

$$
W^{\frac{n}{p}+\varepsilon, p}(\Omega) \times W^{0, p}(\Omega) \longleftrightarrow W^{0, p}(\Omega) . \quad\left(L^{\infty} \times L^{p} \longleftrightarrow L^{p}\right)
$$

Let $\theta=\frac{s}{\frac{n}{p}+\varepsilon}$; clearly $0<\theta<1$. Let $p_{1}=1$ (so if we let $\frac{1}{r}=\frac{1}{p_{1}}+\frac{1}{p}-1$, then $r=p$ ). We want to use real interpolation between (7.1) and (7.2). By Theorem 3.6 we have

$$
\begin{gathered}
\left(W^{\frac{n}{p}+\varepsilon, p}(\Omega), W^{\frac{n}{p}+\varepsilon, p}(\Omega)\right)_{\theta, p_{1}} \times\left(W^{0, p}(\Omega), W^{\frac{n}{p}+\varepsilon, p}(\Omega)\right)_{\theta, p} \\
\longrightarrow\left(W^{0, p}(\Omega), W^{\frac{n}{p}+\varepsilon, p}(\Omega)\right)_{\theta, r} .
\end{gathered}
$$

By Theorem 3.1 we have

$$
\begin{aligned}
\left(W^{\frac{n}{p}+\varepsilon, p}(\Omega), W^{\frac{n}{p}+\varepsilon, p}(\Omega)\right)_{\theta, p_{1}} & =W^{\frac{n}{p}+\varepsilon, p} \\
\left(W^{0, p}(\Omega), W^{\frac{n}{p}+\varepsilon, p}(\Omega)\right)_{\theta, p} & =W^{s, p} . \quad(s \notin \mathbb{Z})
\end{aligned}
$$

Hence

$$
W^{\frac{n}{p}+\varepsilon, p}(\Omega) \times W^{s, p}(\Omega) \longleftrightarrow W^{s, p}(\Omega) .
$$


Theorem 7.3. (Multiplication theorem for Sobolev spaces on the whole space, nonnegative exponents) Assume $s_{i}, s$ and $1 \leq p_{i} \leq p<\infty(i=1,2)$ are real numbers satisfying

(i) $s_{i} \geq s$

(ii) $s \geq 0$,

(iii) $s_{i}-s \geq n\left(\frac{1}{p_{i}}-\frac{1}{p}\right)$,

(iv) $s_{1}+s_{2}-s>n\left(\frac{1}{p_{1}}+\frac{1}{p_{2}}-\frac{1}{p}\right)$.

Claim. If $u \in W^{s_{1}, p_{1}}\left(\mathbb{R}^{n}\right)$ and $v \in W^{s_{2}, p_{2}}\left(\mathbb{R}^{n}\right)$, then $u v \in W^{s, p}\left(\mathbb{R}^{n}\right)$ and moreover the pointwise multiplication of functions is a continuous bilinear map

$$
W^{s_{1}, p_{1}}\left(\mathbb{R}^{n}\right) \times W^{s_{2}, p_{2}}\left(\mathbb{R}^{n}\right) \longrightarrow W^{s, p}\left(\mathbb{R}^{n}\right) .
$$

Proof of Theorem 7.3. First we consider the special case where $p_{1}=p_{2}=p$ and then we will prove the general case.

Step $1 p_{1}=p_{2}=p$ : In this case the assumptions can be rewritten as follows:

$$
s_{1}, s_{2} \geq s \geq 0, \quad s_{1}+s_{2}-s>\frac{n}{p} .
$$

Case $1 s>\frac{n}{p}$ : If $s>\frac{n}{p}$, then $W^{s, p}\left(\mathbb{R}^{n}\right)$ is an algebra and therefore we can write

$$
\begin{aligned}
W^{s_{1}, p_{1}}\left(\mathbb{R}^{n}\right) \times W^{s_{2}, p_{2}}\left(\mathbb{R}^{n}\right) & \longrightarrow W^{s, p}\left(\mathbb{R}^{n}\right) \times W^{s, p}\left(\mathbb{R}^{n}\right) \quad \text { (by assumption } s_{1}, s_{2} \geq s \text { ) } \\
& \longrightarrow W^{s, p}\left(\mathbb{R}^{n}\right) .
\end{aligned}
$$

Case $2 s \leq \frac{n}{p}$ : By Lemma 7.2 for all $\varepsilon>0$

$$
\begin{aligned}
& W^{s, p}\left(\mathbb{R}^{n}\right) \times W^{\frac{n}{p}+\varepsilon, p}\left(\mathbb{R}^{n}\right) \longleftrightarrow W^{s, p}\left(\mathbb{R}^{n}\right), \\
& W^{\frac{n}{p}+\varepsilon, p}\left(\mathbb{R}^{n}\right) \times W^{s, p}\left(\mathbb{R}^{n}\right) \longleftrightarrow W^{s, p}\left(\mathbb{R}^{n}\right) .
\end{aligned}
$$

In particular, for $\varepsilon=s_{1}+s_{2}-s-\frac{n}{p}>0$ we have

$$
\begin{aligned}
& W^{s, p}\left(\mathbb{R}^{n}\right) \times W^{s_{1}+s_{2}-s, p}\left(\mathbb{R}^{n}\right) \longleftrightarrow W^{s, p}\left(\mathbb{R}^{n}\right), \\
& W^{s_{1}+s_{2}-s, p}\left(\mathbb{R}^{n}\right) \times W^{s, p}\left(\mathbb{R}^{n}\right) \longleftrightarrow W^{s, p}\left(\mathbb{R}^{n}\right) .
\end{aligned}
$$

We may consider the following cases:

(i) $p<2, s_{1}, s_{2} \notin \mathbb{Z}$ : Let $\frac{1}{r}=\frac{1}{p}+\frac{1}{p}-1>0$. Let $\theta$ be such that $(1-\theta) s+\theta\left(s_{1}+\right.$ $\left.s_{2}-s\right)=s_{1}$. As it was discussed in the proof of Theorem 5.1, for this $\theta$, (1$\theta)\left(s_{1}+s_{2}-s\right)+\theta s=s_{2}$. By Theorem 3.6 we have

$$
\begin{aligned}
&\left(W^{s, p}\left(\mathbb{R}^{n}\right), W^{s_{1}+s_{2}-s, p}\left(\mathbb{R}^{n}\right)\right)_{\theta, p} \times\left(W^{s_{1}+s_{2}-s, p}\left(\mathbb{R}^{n}\right), W^{s, p}\left(\mathbb{R}^{n}\right)\right)_{\theta, p} \\
& \longleftrightarrow\left(W^{s, p}\left(\mathbb{R}^{n}\right), W^{s, p}\left(\mathbb{R}^{n}\right)\right)_{\theta, r} .
\end{aligned}
$$


Consequently, since $s_{1} \notin \mathbb{Z}$ and $s_{2} \notin \mathbb{Z}$,

$$
W^{s_{1}, p}\left(\mathbb{R}^{n}\right) \times W^{s_{2}, p}\left(\mathbb{R}^{n}\right) \longleftrightarrow W^{s, p}\left(\mathbb{R}^{n}\right) .
$$

(ii) $p<2, s_{1} \in \mathbb{Z}, s_{2} \notin \mathbb{Z}$ : If $s_{1}=s$, then from $s_{1}+s_{2}-s>\frac{n}{p}$ it follows that $s_{2}>\frac{n}{p}$. So, in this case the claim reduces to what was proved in Lemma 7.2. If $s_{1} \neq s$, let $\tilde{s}_{1}=s_{1}-\varepsilon$ where

$$
\varepsilon=\frac{1}{2} \min \left(s_{1}-\left\lfloor s_{1}\right\rfloor, s_{1}-s, s_{1}+s_{2}-s-\frac{n}{p}\right)>0 .
$$

Clearly,

$$
\tilde{s}_{1} \notin \mathbb{Z}, \quad \tilde{s}_{1} \geq s, \quad s_{2} \geq s, \quad \tilde{s}_{1}+s_{2}-s>\frac{n}{p} .
$$

Therefore, by what was proved in the previous case

$$
W^{\tilde{s}_{1}, p} \times W^{s_{2}, p} \longleftrightarrow W^{s, p} .
$$

Now the claim follows from the fact that $W^{s_{1}, p} \hookrightarrow W^{\tilde{s}_{1}, p}$.

(iii) $p<2, s_{1} \notin \mathbb{Z}, s_{2} \in \mathbb{Z}$ : Just switch the roles of $s_{1}$ and $s_{2}$ in the previous case.

(iv) $p<2, s_{1} \in \mathbb{Z}, s_{2} \in \mathbb{Z}$ : Note that both of $s_{1}$ and $s_{2}$ cannot be equal to $s$ because $s_{1}+s_{2}-s>\frac{n}{p}$ but $s \leq \frac{n}{p}$. Because of the symmetry in the roles of $s_{1}$ and $s_{2}$, without loss of generality we may assume that $s_{1} \neq s$. Let $\tilde{s}_{1}=s_{1}-\varepsilon$ where

$$
\varepsilon=\frac{1}{2} \min \left(s_{1}-\left\lfloor s_{1}\right\rfloor, s_{1}-s, s_{1}+s_{2}-s-\frac{n}{p}\right)>0 .
$$

Clearly,

$$
\tilde{s}_{1} \notin \mathbb{Z}, \quad \tilde{s}_{1} \geq s, \quad s_{2} \geq s, \quad \tilde{s}_{1}+s_{2}-s>\frac{n}{p} .
$$

and so the problem reduces to the previous case.

At this point we are done with the case $p<2$.

(v) $p \geq 2, s \notin \mathbb{Z}, s_{1}+s_{2}-s \notin \mathbb{Z}$ : This time we use complex interpolation. Define $\theta$ as before. By Theorem 3.6 we have

$$
\begin{aligned}
{\left[W^{s, p}\left(\mathbb{R}^{n}\right), W^{s_{1}+s_{2}-s, p}\left(\mathbb{R}^{n}\right)\right]_{\theta} \times\left[W^{s_{1}+s_{2}-s, p}\left(\mathbb{R}^{n}\right), W^{s, p}\left(\mathbb{R}^{n}\right)\right]_{\theta} } & \\
& \longleftrightarrow\left[W^{s, p}\left(\mathbb{R}^{n}\right), W^{s, p}\left(\mathbb{R}^{n}\right)\right]_{\theta} .
\end{aligned}
$$

Since $s$ and $s_{1}+s_{2}-s$ are not integers and $p \geq 2$ (see Theorem 3.2),

$$
\begin{aligned}
& W^{s_{1}, p}\left(\mathbb{R}^{n}\right) \longleftrightarrow\left[W^{s, p}\left(\mathbb{R}^{n}\right), W^{s_{1}+s_{2}-s, p}\left(\mathbb{R}^{n}\right)\right]_{\theta}, \\
& W^{s_{2}, p}\left(\mathbb{R}^{n}\right) \longleftrightarrow\left[W^{s_{1}+s_{2}-s, p}\left(\mathbb{R}^{n}\right), W^{s, p}\left(\mathbb{R}^{n}\right)\right]_{\theta} .
\end{aligned}
$$

Consequently,

$$
W^{s_{1}, p}\left(\mathbb{R}^{n}\right) \times W^{s_{2}, p}\left(\mathbb{R}^{n}\right) \longleftrightarrow W^{s, p}\left(\mathbb{R}^{n}\right) .
$$


(vi) $p \geq 2, s \notin \mathbb{Z}, s_{1}+s_{2}-s \in \mathbb{Z}$ : Both of $s_{1}$ and $s_{2}$ cannot be equal to $s$ because $s_{1}+s_{2}-s>\frac{n}{p}$ but $s \leq \frac{n}{p}$. Because of the symmetry in the roles of $s_{1}$ and $s_{2}$, without loss of generality we may assume that $s_{1} \neq s$. Let $\tilde{s}_{1}=s_{1}-\varepsilon$ where

$$
\varepsilon=\frac{1}{2} \min \left(1, s_{1}-s, s_{1}+s_{2}-s-\frac{n}{p}\right) .
$$

Clearly,

$$
\begin{aligned}
& \tilde{s}_{1} \geq s, \quad \tilde{s}_{1}+s_{2}-s=s_{1}+s_{2}-s-\varepsilon>\frac{n}{p}, \\
& \tilde{s}_{1}+s_{2}-s=s_{1}+s_{2}-s-\varepsilon \notin \mathbb{Z} \quad\left(\text { because } \varepsilon \leq \frac{1}{2}\right) .
\end{aligned}
$$

So, by what was proved in the previous case we have

$$
W^{\tilde{s}_{1}, p}\left(\mathbb{R}^{n}\right) \times W^{s_{2}, p}\left(\mathbb{R}^{n}\right) \longleftrightarrow W^{s, p}\left(\mathbb{R}^{n}\right),
$$

and since $W^{s_{1}, p_{\hookrightarrow}} W^{\tilde{s}_{1}, p}$,

$$
W^{s_{1}, p}\left(\mathbb{R}^{n}\right) \times W^{s_{2}, p}\left(\mathbb{R}^{n}\right) \longleftrightarrow W^{s, p}\left(\mathbb{R}^{n}\right) .
$$

(vii) $p \geq 2, s \in \mathbb{Z}, s_{1}+s_{2}-s \notin \mathbb{Z}$ : If $s_{1}=s$ or $s_{2}=s$, the claim follows from Lemma 7.2. So we may assume $s_{1}, s_{2}>s$. Let $\tilde{s}=s+\varepsilon$ where

$$
\begin{aligned}
& \varepsilon=\frac{1}{2} \min \left(s_{1}-s, s_{2}-s, s_{1}+s_{2}-s-\left\lfloor s_{1}+s_{2}-s\right\rfloor, s_{1}+s_{2}-s-\frac{n}{p}\right) \\
& \text { (note that } s_{1}+s_{2}-s-\left\lfloor s_{1}+s_{2}-s\right\rfloor<1 \text { ) }
\end{aligned}
$$

Clearly $\tilde{s}$ and $s_{1}+s_{2}-\tilde{s}$ are not integers and

$$
s_{1} \geq \tilde{s}, \quad s_{2} \geq \tilde{s}, \quad s_{1}+s_{2}-\tilde{s}>\frac{n}{p} .
$$

So, by what was proved in previous cases

$$
W^{s_{1}, p}\left(\mathbb{R}^{n}\right) \times W^{s_{2}, p}\left(\mathbb{R}^{n}\right) \longleftrightarrow W^{\tilde{s}, p}\left(\mathbb{R}^{n}\right)=W^{s+\varepsilon, p}\left(\mathbb{R}^{n}\right) \longleftrightarrow W^{s, p}\left(\mathbb{R}^{n}\right) .
$$

(viii) $p \geq 2, s \in \mathbb{Z}, s_{1}+s_{2}-s \in \mathbb{Z}$ : If $s_{1}=s$ or $s_{2}=s$, the claim follows from Lemma 7.2. So we may assume $s_{1}, s_{2}>s$. Let $\tilde{s}=s+\varepsilon$ where

$$
\varepsilon=\frac{1}{2}\left(1, s_{1}-s, s_{2}-s, s_{1}+s_{2}-s-\frac{n}{p}\right) .
$$

We have $\varepsilon \leq \frac{1}{2}$, so $\tilde{s}$ and $s_{1}+s_{2}-\tilde{s}$ are not integers. Also, clearly

$$
s_{1} \geq \tilde{s}, \quad s_{2} \geq \tilde{s}, \quad s_{1}+s_{2}-\tilde{s}>\frac{n}{p} .
$$

So, by what was proved in previous cases

$$
W^{s_{1}, p}\left(\mathbb{R}^{n}\right) \times W^{s_{2}, p}\left(\mathbb{R}^{n}\right) \longleftrightarrow W^{\tilde{s}, p}\left(\mathbb{R}^{n}\right)=W^{s+\varepsilon, p}\left(\mathbb{R}^{n}\right) \longleftrightarrow W^{s, p}\left(\mathbb{R}^{n}\right) .
$$


Step 2: General Case. This step is exactly the same as Step 2 in the proof of Theorem 5.1. We just need to replace every occurrence of $H^{r, q}\left(\mathbb{R}^{n}\right)$ with $W^{r, q}\left(\mathbb{R}^{n}\right)$.

Proposition 4.3 shows that the claim of Theorem 7.3 does not necessarily hold if one removes the assumption $p_{i} \leq p$. Of course, the next theorem shows that the assumption $p_{i} \leq p$ is not necessary on bounded domains.

Theorem 7.4. (Multiplication theorem for Sobolev spaces on bounded domains, nonnegative exponents) Let $\Omega$ be a bounded domain in $\mathbb{R}^{n}$ with Lipschitz continuous boundary. Assume $s_{i}, s$ and $1 \leq p_{i}, p<\infty(i=1,2)$ are real numbers satisfying

(i) $s_{i} \geq s$

(ii) $s \geq 0$,

(iii) $s_{i}-s \geq n\left(\frac{1}{p_{i}}-\frac{1}{p}\right)$,

(iv) $s_{1}+s_{2}-s>n\left(\frac{1}{p_{1}}+\frac{1}{p_{2}}-\frac{1}{p}\right)$.

In the case where $\max \left\{p_{1}, p_{2}\right\}>p$ instead of $(i v)$ assume that $s_{1}+s_{2}-s>\frac{n}{\min \left\{p_{1}, p_{2}\right\}}$ and that the inequalities in $(i)$ and (iii) are strict.

Claim: If $u \in W^{s_{1}, p_{1}}(\Omega)$ and $v \in W^{s_{2}, p_{2}}(\Omega)$, then $u v \in W^{s, p}(\Omega)$ and moreover the pointwise multiplication of functions is a continuous bilinear map

$$
W^{s_{1}, p_{1}}(\Omega) \times W^{s_{2}, p_{2}}(\Omega) \longrightarrow W^{s, p}(\Omega) .
$$

Proof of Theorem 7.4.

Step $1 p_{1}=p_{2}=p$ : By the (exact) same proof as the one given in Step 1 of the proof of Theorem 7.3 we have

$$
W^{s_{1}, p}(\Omega) \times W^{s_{2}, p}(\Omega) \longleftrightarrow W^{s, p}(\Omega),
$$

provided $s_{1}, s_{2} \geq s$ and $s_{1}+s_{2}-s>\frac{n}{p}$.

Step 2: Now we prove the general case. Because of the symmetry in the roles of $p_{1}$ and $p_{2}$ without loss of generality we may assume $p_{2} \leq p_{1}$. We may consider three cases:

Case $1 p_{2} \leq p_{1} \leq p$ : The proof is exactly the same as the one presented in Step 2 of Theorem 7.3.

Case $2 p_{2} \leq p<p_{1}$ : Let $\tilde{p}_{1}=p$. Since, by assumption, $s_{1}+s_{2}-s>\frac{n}{p_{2}}$, we can choose $\tilde{s}_{1} \in\left(s, s_{1}\right)$ such that $\tilde{s}_{1}+s_{2}-s>\frac{n}{p_{2}}$. Now, one can easily check that the tuple $\left(\tilde{s}_{1}, s_{2}, s, \tilde{p}_{1}, p_{2}, p\right)$ also satisfies all the assumptions of the theorem. So, by what was proved in the previous case we have

$$
W^{\tilde{s}_{1}, \tilde{p}_{1}}(\Omega) \times W^{s_{2}, p_{2}}(\Omega) \longleftrightarrow W^{s, p}(\Omega) .
$$


By the third embedding theorem (Theorem 3.10) $W^{s_{1}, p_{1}}(\Omega) \hookrightarrow W^{\tilde{s}_{1}, \tilde{p}_{1}}(\Omega)$ (because $\left.s_{1}-\tilde{s}_{1}>0>\frac{n}{p_{1}}-\frac{n}{\tilde{p}_{1}}\right)$. Hence

$$
W^{s_{1}, p_{1}}(\Omega) \times W^{s_{2}, p_{2}}(\Omega) \longleftrightarrow W^{s, p}(\Omega) .
$$

Case $3 p<p_{2} \leq p_{1}$ : Let

$$
\varepsilon=\frac{1}{6} \min \left\{s_{1}-s, s_{2}-s, s_{1}+s_{2}-s-\frac{n}{p_{2}}\right\}
$$

and set $\hat{s}_{1}=s_{1}-\varepsilon, \hat{s}_{2}=s_{2}-\varepsilon$, and $\hat{s}=s+\varepsilon$. Clearly, $\hat{s}_{1}, \hat{s}_{2} \geq \hat{s}$. Moreover, Since $s_{1}+s_{2}-s>\frac{n}{p_{2}}$, we have

$$
\hat{s}_{1}+\hat{s}_{2}-\hat{s}=s_{1}+s_{2}-s-3 \varepsilon>\frac{n}{p_{2}}
$$

Thus, by what was proved in Step 1 we have

$$
W^{\hat{s}_{1}, p_{2}}(\Omega) \times W^{\hat{s}_{2}, p_{2}}(\Omega) \longleftrightarrow W^{\hat{s}, p_{2}}(\Omega) .
$$

Now, note that $p_{1} \geq p_{2}$ and $p_{2}>p$, so by the third embedding theorem (Theorem 3.10) $W^{s_{1}, p_{1}}(\Omega) \hookrightarrow W^{\hat{s}_{1}, p_{2}}(\Omega)$ and $W^{\hat{s}, p_{2}}(\Omega) \hookrightarrow W^{s, p}(\Omega)$. Therefore,

$$
W^{s_{1}, p_{1}}(\Omega) \times W^{s_{2}, p_{2}}(\Omega) \longleftrightarrow W^{s, p}(\Omega) .
$$

\section{Sufficient conditions for $W^{s_{1}, p_{1}} \times W^{s_{2}, p_{2}} \hookrightarrow W^{s, p}, s<0, s \in \mathbb{R}$}

Theorem 8.1. (Multiplication theorem for Sobolev spaces on the whole space, negative exponents I) Assume $s_{i}, s$ and $1<p_{i} \leq p<\infty(i=1,2)$ are real numbers satisfying

(i) $s_{i} \geq s$,

(ii) $\min \left\{s_{1}, s_{2}\right\}<0$,

(iii) $s_{i}-s \geq n\left(\frac{1}{p_{i}}-\frac{1}{p}\right)$,

(iv) $s_{1}+s_{2}-s>n\left(\frac{1}{p_{1}}+\frac{1}{p_{2}}-\frac{1}{p}\right)$.

(v) $s_{1}+s_{2} \geq n\left(\frac{1}{p_{1}}+\frac{1}{p_{2}}-1\right) \geq 0$.

Then the pointwise multiplication of functions extends uniquely to a continuous bilinear map

$$
W^{s_{1}, p_{1}}\left(\mathbb{R}^{n}\right) \times W^{s_{2}, p_{2}}\left(\mathbb{R}^{n}\right) \longrightarrow W^{s, p}\left(\mathbb{R}^{n}\right)
$$

Proof of Theorem 8.1. Since by assumption $s_{1}+s_{2} \geq 0, s_{1}$ and $s_{2}$ cannot both be negative. Without loss of generality we can assume $s_{1}$ is negative and $s_{2}$ is positive. Also note that by assumption $s \leq s_{1}$ so $s$ is also negative. 
Note that $C_{c}^{\infty}$ is dense in all Sobolev spaces on $\mathbb{R}^{n}$. Considering this, first we prove that for $u \in W^{s_{1}, p_{1}}, \varphi \in C_{c}^{\infty}$

$$
\|u \varphi\|_{s, p} \preceq\|u\|_{s_{1}, p_{1}}\|\varphi\|_{s_{2}, p_{2}} .
$$

Note that

$$
\|f\|_{s, p}=\sup _{\psi \in C_{c}^{\infty}} \frac{\left|\langle f, \psi\rangle_{W^{s, p} \times W^{-s, p^{\prime}}}\right|}{\|\psi\|_{-s, p^{\prime}}} .
$$

Thus we just need to show that

$$
\left|\langle u \varphi, \psi\rangle_{W^{s, p} \times W^{-s, p^{\prime}}}\right| \preceq\|u\|_{s_{1}, p_{1}}\|\varphi\|_{s_{2}, p_{2}}\|\psi\|_{-s, p^{\prime}} .
$$

We have

$$
\left|\langle u \varphi, \psi\rangle_{W^{s, p} \times W^{-s, p^{\prime}}}\right|=\left|\langle u, \varphi \psi\rangle_{W^{s_{1}, p_{1}} \times W^{-s_{1}, p_{1}^{\prime}}}\right| \preceq\|u\|_{s_{1}, p_{1}}\|\varphi \psi\|_{-s_{1}, p_{1}^{\prime}} .
$$

Note that the first equality holds true because duality pairing is an extension of the $L^{2}$ inner product of smooth functions. Now, it is enough to prove that

$$
\|\varphi \psi\|_{-s_{1}, p_{1}^{\prime}} \preceq\|\varphi\|_{s_{2}, p_{2}}\|\psi\|_{-s, p^{\prime}} .
$$

$-s_{1}, s_{2},-s$ are all nonnegative. So, by Theorem 7.3 , in order to ensure that the above inequality is true we just need to check the followings:

$p^{\prime} \leq p_{1}^{\prime} \quad\left(\right.$ true because $\left.p_{1} \leq p\right), \quad p_{2} \leq p_{1}^{\prime} \quad\left(\right.$ true because $\left.\frac{1}{p_{1}}+\frac{1}{p_{2}} \geq 1\right)$,

$-s_{1} \leq-s \quad\left(\right.$ true because $\left.s \leq s_{1}\right), \quad s_{2} \geq-s_{1} \quad\left(\right.$ true because $\left.s_{1}+s_{2} \geq 0\right)$,

$s_{2}-\left(-s_{1}\right) \geq n\left(\frac{1}{p_{2}}-\frac{1}{p_{1}^{\prime}}\right) \quad\left(\right.$ true because $\left.s_{2}+s_{1} \geq n\left(\frac{1}{p_{2}}+\frac{1}{p_{1}}-1\right)\right)$,

$-s-\left(-s_{1}\right) \geq n\left(\frac{1}{p^{\prime}}-\frac{1}{p_{1}^{\prime}}\right) \quad\left(\right.$ true because $\left.s_{1}-s \geq n\left(\frac{1}{p_{1}}-\frac{1}{p}\right)\right)$,

$s_{2}+(-s)-\left(-s_{1}\right)>n\left(\frac{1}{p_{2}}+\frac{1}{p^{\prime}}-\frac{1}{p_{1}^{\prime}}\right) \quad\left(\right.$ true because $\left.s_{1}+s_{2}-s>n\left(\frac{1}{p_{2}}+\frac{1}{p_{1}}-\frac{1}{p}\right)\right)$.

Therefore, the inequality (8.1) holds for $u \in W^{s_{1}, p_{1}}$ and $\varphi \in C_{c}^{\infty}$. To prove the general case we proceed as follows: Suppose $v \in W^{s_{2}, p_{2}}$. There exists a sequence $\varphi_{k} \in C_{c}^{\infty}$ such that $\varphi_{k} \rightarrow v$ in $W^{s_{2}, p_{2}}$. Since $s_{2} \geq 0, W^{s_{2}, p_{2}} \hookrightarrow L^{p_{2}}$ and therefore $\varphi_{k} \rightarrow v$ in $L^{p_{2}}$. Consequently, by possibly passing to a subsequence, $\varphi_{k} \rightarrow v$ a.e. which implies that $u \varphi_{k} \rightarrow u v$ a.e..

On the other hand we have

$$
\left\|u\left(\varphi_{i}-\varphi_{j}\right)\right\|_{s, p} \preceq\|u\|_{s_{1}, p_{1}}\left\|\varphi_{i}-\varphi_{j}\right\|_{s_{2}, p_{2}} .
$$


It follows that $u \varphi_{k}$ is a Cauchy sequence in $W^{s, p}$ and thus it is convergent to some function $w \in W^{s, p}$. Since $u \varphi_{k} \rightarrow u v$ a.e., we can conclude that $w=u v$, that is, $u \varphi_{k} \rightarrow u v$ in $W^{s, p}$. Finally

$$
\forall k \quad\left\|u \varphi_{k}\right\|_{s, p} \preceq\|u\|_{s_{1}, p_{1}}\left\|\varphi_{k}\right\|_{s_{2}, p_{2}},
$$

and so by passing to the limit as $k \rightarrow \infty$

$$
\|u v\|_{s, p} \preceq\|u\|_{s_{1}, p_{1}}\|v\|_{s_{2}, p_{2}} .
$$

Theorem 8.2. (Multiplication theorem for Sobolev spaces on the whole space, negative exponents II) Assume $s_{i}, s$ and $1<p, p_{i}<\infty(i=1,2)$ are real numbers satisfying

(i) $s_{i} \geq s$,

(ii) $\min \left\{s_{1}, s_{2}\right\} \geq 0$ and $s<0$,

(iii) $s_{i}-s \geq n\left(\frac{1}{p_{i}}-\frac{1}{p}\right)$,

(iv) $s_{1}+s_{2}-s>n\left(\frac{1}{p_{1}}+\frac{1}{p_{2}}-\frac{1}{p}\right) \geq 0$.

(v) $s_{1}+s_{2}>n\left(\frac{1}{p_{1}}+\frac{1}{p_{2}}-1\right)$. (the inequality is strict)

Then the pointwise multiplication of functions extends uniquely to a continuous bilinear map

$$
W^{s_{1}, p_{1}}\left(\mathbb{R}^{n}\right) \times W^{s_{2}, p_{2}}\left(\mathbb{R}^{n}\right) \longrightarrow W^{s, p}\left(\mathbb{R}^{n}\right)
$$

Proof of Theorem 8.2. Let $\varepsilon>0$ be such that

$$
\varepsilon<\frac{1}{n} \min \left\{s_{1}+s_{2}-s-\left(\frac{n}{p_{1}}+\frac{n}{p_{2}}-\frac{n}{p}\right), s_{1}+s_{2}-\left(\frac{n}{p_{1}}+\frac{n}{p_{2}}-n\right)\right\} .
$$

Let

$$
\frac{1}{r}=\max \left\{\frac{1}{p_{1}}-\frac{s_{1}}{n}, \frac{1}{p_{2}}-\frac{s_{2}}{n}, \frac{1}{p_{1}}-\frac{s_{1}}{n}+\frac{1}{p_{2}}-\frac{s_{2}}{n}+\varepsilon, \frac{1}{p}\right\} .
$$

Note that $r>0$ because $\frac{1}{r} \geq \frac{1}{p}>0$. Also $\frac{1}{r}<1$ because each element in the set over which we are taking the maximum is strictly less than 1 :

$$
\begin{aligned}
& \frac{1}{p_{1}}-\frac{s_{1}}{n} \leq \frac{1}{p_{1}}<1, \quad \frac{1}{p_{2}}-\frac{s_{2}}{n} \leq \frac{1}{p_{2}}<1, \quad \frac{1}{p}<1 \\
& \varepsilon<\frac{1}{n}\left[s_{1}+s_{2}-\left(\frac{n}{p_{1}}+\frac{n}{p_{2}}-n\right)\right] \Longrightarrow \frac{1}{p_{1}}-\frac{s_{1}}{n}+\frac{1}{p_{2}}-\frac{s_{2}}{n}+\varepsilon<1 .
\end{aligned}
$$

Claim 1: $W^{s_{1}, p_{1}}\left(\mathbb{R}^{n}\right) \times W^{s_{2}, p_{2}}\left(\mathbb{R}^{n}\right) \hookrightarrow L^{r}\left(\mathbb{R}^{n}\right)$.

Claim 2: $L^{r}\left(\mathbb{R}^{n}\right) \hookrightarrow W^{s, p}\left(\mathbb{R}^{n}\right)$.

Clearly if we prove Claim 1 and Claim 2, then we are done. 
Proof of Claim 1. All the exponents are nonnegative, so it is enough to check the assumptions of Theorem 6.1 .

$$
\begin{aligned}
& s_{1}-0 \geq n\left(\frac{1}{p_{1}}-\frac{1}{r}\right) \quad\left(\text { true because } \frac{1}{r} \geq \frac{1}{p_{1}}-\frac{s_{1}}{n}\right) \\
& s_{2}-0 \geq n\left(\frac{1}{p_{2}}-\frac{1}{r}\right) \quad\left(\text { true because } \frac{1}{r} \geq \frac{1}{p_{2}}-\frac{s_{2}}{n}\right) \\
& s_{1}+s_{2}>n\left(\frac{1}{p_{1}}+\frac{1}{p_{2}}-\frac{1}{r}\right) \quad\left(\text { true because } \frac{1}{r}>\frac{1}{p_{1}}-\frac{s_{1}}{n}+\frac{1}{p_{2}}-\frac{s_{2}}{n}\right) .
\end{aligned}
$$

Proof of Claim 2. We have $\left(L^{r}\left(\mathbb{R}^{n}\right)\right)^{*}=L^{r^{\prime}}\left(\mathbb{R}^{n}\right) \quad$ and $\quad\left(W^{s, p}\left(\mathbb{R}^{n}\right)\right)^{*}=$ $W^{-s, p^{\prime}}\left(\mathbb{R}^{n}\right)$. In what follows we will show that $W^{-s, p^{\prime}}\left(\mathbb{R}^{n}\right) \hookrightarrow L^{r^{\prime}}\left(\mathbb{R}^{n}\right)$; then since $W^{-s, p^{\prime}}\left(\mathbb{R}^{n}\right)$ is dense in $L^{r^{\prime}}\left(\mathbb{R}^{n}\right)\left(C_{c}^{\infty}\left(\mathbb{R}^{n}\right) \subseteq W^{-s, p^{\prime}}\left(\mathbb{R}^{n}\right)\right.$ and $C_{c}^{\infty}\left(\mathbb{R}^{n}\right)$ is dense in $\left.L^{r^{\prime}}\left(\mathbb{R}^{n}\right)\right)$, we are allowed to take the dual of both sides and it immediately follows that the claim is true.

Note that by the definition of $r$, we have $\frac{1}{p} \leq \frac{1}{r}$ and therefore $p^{\prime} \leq r^{\prime}$. So, according to Theorem 3.8, in order to show that $W^{-s, p^{\prime}}\left(\mathbb{R}^{n}\right) \hookrightarrow L^{r^{\prime}}\left(\mathbb{R}^{n}\right)$, it is enough to prove that $-s-\frac{n}{p^{\prime}} \geq 0-\frac{n}{r^{\prime}}$, that is, we need to prove that $\frac{1}{p}-\frac{s}{n} \geq \frac{1}{r}$. This is true because each element in the set over which we are taking the maximum in the definition of $\frac{1}{r}$ is less than or equal to $\frac{1}{p}-\frac{s}{n}$ :

$$
\begin{aligned}
& \frac{1}{p}-\frac{s}{n} \geq \frac{1}{p_{1}}-\frac{s_{1}}{n} \quad\left(\text { true because } s_{1}-s \geq n\left(\frac{1}{p_{1}}-\frac{1}{p}\right)\right) \\
& \frac{1}{p}-\frac{s}{n} \geq \frac{1}{p_{2}}-\frac{s_{2}}{n} \quad\left(\text { true because } s_{2}-s \geq n\left(\frac{1}{p_{2}}-\frac{1}{p}\right)\right) \\
& \frac{1}{p}-\frac{s}{n} \geq \frac{1}{p_{1}}-\frac{s_{1}}{n}+\frac{1}{p_{2}}-\frac{s_{2}}{n}+\varepsilon \quad\left(\text { true because } s_{1}+s_{2}-s \geq n\left(\frac{1}{p_{1}}+\frac{1}{p_{2}}-\frac{1}{p}\right)+n \varepsilon\right) \\
& \frac{1}{p}-\frac{s}{n} \geq \frac{1}{p} \quad(\text { true because } s<0)
\end{aligned}
$$

This completes the proof of the Theorem.

Remark 8.3. We note that our earlier article [8] contains some multiplication results for negative exponents that are similar to what we give above as Theorem 8.2. However, a particularly important case is assumption (ii) in Theorem 8.2 above, which is a case we did not consider in [8].

Acknowledgments. MH was supported in part by NSF Awards 1262982, 1318480, and 1620366. AB was supported by NSF Award 1262982. 


\section{A. A useful version of the multiplication theorem}

In this appendix, using the pointwise multiplication properties of Besov spaces, we will prove a very useful multiplication theorem in Sobolev-Slobodeckij spaces; this multiplication result is a key tool used in our related article [3] to study the Einstein constraint equations on asymptotically flat manifolds. A similar result, but for the case of compact manifolds, was used in our 2009 article [8]. We include this result to help complete the supporting literature for the work in both [3] and [8].

Theorem A.1. Let $s_{i} \geq s$ with $s_{1}+s_{2} \geq 0$, and $1<p, p_{i}<\infty(i=1,2)$ be real numbers satisfying

$$
\begin{aligned}
& s_{i}-s \geq n\left(\frac{1}{p_{i}}-\frac{1}{p}\right), \quad\left(\text { if } s_{i}=s \notin \mathbb{Z}, \text { then let } p_{i} \leq p\right) \\
& s_{1}+s_{2}-s>n\left(\frac{1}{p_{1}}+\frac{1}{p_{2}}-\frac{1}{p}\right) \geq 0 .
\end{aligned}
$$

In case $s<0$, in addition let

$$
s_{1}+s_{2}>n\left(\frac{1}{p_{1}}+\frac{1}{p_{2}}-1\right) \quad\left(\text { equality is allowed if } \min \left(s_{1}, s_{2}\right)<0\right) .
$$

Also in case where $s_{1}+s_{2}=0$ and $\min \left(s_{1}, s_{2}\right) \notin \mathbb{Z}$, in addition let $\frac{1}{p_{1}}+\frac{1}{p_{2}} \geq 1$. Then the pointwise multiplication of functions extends uniquely to a continuous bilinear map

$$
W^{s_{1}, p_{1}}\left(\mathbb{R}^{n}\right) \times W^{s_{2}, p_{2}}\left(\mathbb{R}^{n}\right) \longrightarrow W^{s, p}\left(\mathbb{R}^{n}\right)
$$

Proof of Theorem A.1. In this proof we use the notations introduced in the beginning of Section 4 . We may consider three cases:

Case 1: $s \geq 0$.

Case 2: $s<0$ and $\min \left(s_{1}, s_{2}\right)<0$.

Case 3: $s<0$ and $\min \left(s_{1}, s_{2}\right) \geq 0$.

In what follows we study each of the above cases separately.

- Case 1: See Theorem 6.1 for the case where $s \in \mathbb{N}_{0}$; see Theorem 7.3 for the case where $p_{1}, p_{2} \leq p$. It remains to prove the claim in the following cases:

i. $s_{1}>s, s_{2}=s, s \notin \mathbb{N}_{0}, p_{1}>p, p_{2} \leq p$

ii. $s_{1}=s, s_{2}>s, s \notin \mathbb{N}_{0}, p_{1} \leq p, p_{2}>p$

iii. $s_{1}>s, s_{2}>s, s \notin \mathbb{N}_{0}$, at least one of $p_{1}$ or $p_{2}$ is greater than $p$

Proofs of [i] and [ii] are completely similar. Here we only prove item [i] and item [iii]. 
Proof of $[i]$. Let

$$
\varepsilon:=\frac{1}{4} \min \left\{s_{1}-s, s_{1}-s-n\left(\frac{1}{p_{1}}-\frac{1}{p}\right), s_{1}-n\left(\frac{1}{p_{1}}+\frac{1}{p_{2}}-\frac{1}{p}\right)\right\} .
$$

We have

$$
\begin{aligned}
W^{s_{1}, p_{1}} \times W^{s_{2}, p_{2}} \longleftrightarrow B_{p_{1}, p_{1}}^{s_{1}-\varepsilon} \times W^{s_{2}, p_{2}} \hookrightarrow B_{p_{1}, p}^{s_{1}-\varepsilon} \times W^{s_{2}, p_{2}} \\
=B_{p_{1}, p}^{s_{1}-\varepsilon} \times B_{p_{2}, p_{2}}^{s_{2}} \longleftrightarrow B_{p, p}^{s}=W^{s, p} .
\end{aligned}
$$

Proof of [iii]. Let

$$
\begin{aligned}
\varepsilon:=\frac{1}{4} \min \left\{s_{1}-s, s_{2}-s, s_{1}-s-n\left(\frac{1}{p_{1}}-\frac{1}{p}\right),\right. & s_{2}-s-n\left(\frac{1}{p_{2}}-\frac{1}{p}\right), \\
& \left.s_{1}+s_{2}-s-n\left(\frac{1}{p_{1}}+\frac{1}{p_{2}}-\frac{1}{p}\right)\right\} .
\end{aligned}
$$

Let $\tilde{q}_{1}, \tilde{q}_{2}$, and $\tilde{q}$ be numbers in $(1, \infty)$ such that $\tilde{q}_{1}, \tilde{q}_{2} \leq \tilde{q}$. We have $W^{s_{1}, p_{1}} \times W^{s_{2}, p_{2}} \longleftrightarrow B_{p_{1}, p_{1}}^{s_{1}-\frac{\varepsilon}{2}} \times B_{p_{2}, p_{2}}^{s_{2}-\frac{\varepsilon}{2}} \longleftrightarrow B_{p_{1}, \tilde{q}_{1}}^{s_{1}-\varepsilon} \times B_{p_{2}, \tilde{q}_{2}}^{s_{2}-\varepsilon} \longleftrightarrow B_{p, \tilde{q}}^{s+\varepsilon} \longleftrightarrow B_{p, p}^{s}=W^{s, p}$.

In the above we have used the well-known embedding theorems for Besov spaces together with the following multiplication theorem that is proved in [12]:

Let $0 \leq s \leq s_{i}, 1<p_{i}, p<\infty, 1<q_{i} \leq q<\infty(i=1,2)$ be such that

$$
\begin{aligned}
& s_{i}-s \geq n\left(\frac{1}{p_{i}}-\frac{1}{p}\right), \quad i=1,2, \\
& s_{1}+s_{2}-s>n\left(\frac{1}{p_{1}}+\frac{1}{p_{2}}-\frac{1}{p}\right) .
\end{aligned}
$$

Then for $s \notin \mathbb{N}$ one has $B_{p_{1}, q_{1}}^{s_{1}} \times B_{p_{2}, q_{2}}^{s_{2}} \hookrightarrow B_{p, q}^{s}$.

- Case 2: It follows from the argument given in the proof of Theorem 8.1 that without loss of generality we may assume $s_{1}<0$ and $s_{2}>0$. According to the same argument it is enough to prove that

$$
W^{s_{2}, p_{2}} \times W^{-s, p^{\prime}} \longleftrightarrow W^{-s_{1}, p_{1}^{\prime}}
$$

Since $s_{2},-s$, and $-s_{1}$ are all nonnegative numbers, we can use what was shown in Case 1 to prove the above embedding. We have

。 $s_{1}+s_{2} \geq 0 \Longrightarrow s_{2} \geq-s_{1}$.

० $s_{1} \geq s \Longrightarrow-s \geq-s_{1}$.

。 If $s_{2}=-s_{1}$ (that is, if $s_{1}+s_{2}=0$ ) and $-s_{1} \notin \mathbb{N}$, we must have $p_{2} \leq p_{1}^{\prime}$, i.e., $1 \leq \frac{1}{p_{1}}+\frac{1}{p_{2}}$. (holds true by assumption) 
○If $-s=-s_{1}$ and $-s_{1} \notin \mathbb{N}$, we must have $p^{\prime} \leq p_{1}^{\prime}$, i.e., $p_{1} \leq p$. (holds true by assumption)

$$
\begin{aligned}
& \circ s_{2}+s_{1} \geq n\left(\frac{1}{p_{1}}+\frac{1}{p_{2}}-1\right)=n\left(\frac{1}{p_{2}}-\frac{1}{p_{1}^{\prime}}\right) . \\
& \circ-s+s_{1} \geq n\left(\frac{1}{p_{1}}-\frac{1}{p}\right)=n\left(\frac{1}{p^{\prime}}-\frac{1}{p_{1}^{\prime}}\right) . \\
& \circ s_{2}-s+s_{1}>n\left(\frac{1}{p_{1}}+\frac{1}{p_{2}}-\frac{1}{p}\right)=n\left(\frac{1}{p_{2}}+\frac{1}{p^{\prime}}-\frac{1}{p_{1}^{\prime}}\right) .
\end{aligned}
$$

So, according to what was proved in Case 1, the embedding (A.1) holds true.

- For Case 3, see Theorem 8.2.

Remark A.2. Note that in case $s_{i}=s \notin \mathbb{Z}$, the condition $p_{i} \leq p$ together with $s_{i}-s \geq n\left(\frac{1}{p_{i}}-\frac{1}{p}\right)$ in fact implies that we must have $p_{i}=p$.

\section{References}

1. Adams, R. A. and Fournier, J. J. F., Sobolev Spaces, 2nd ed., Academic Press, New York, 2003.

2. Amann, H., Multiplication in Sobolev and Besov spaces, in Nonlinear Analysis, A Tribute in Honour of G. Prodi, pp. 27-50, Quaderni, Scuola Norm. Sup., Pisa, 1991.

3. Behzadan, A. and Holst, M., Rough solutions of the Einstein constraint equations on asymptotically flat manifolds without near-cmc conditions, Preprint, 2015. arXiv:1504.04661.

4. Bhattacharyya, P. K., Distributions: Generalized functions with applications in Sobolev spaces, de Gruyter, Berlin, 2012.

5. Brezis, H. and Mironescu, P., Gagliardo-Nirenberg, composition and products in fractional Sobolev spaces, J. Evol. Equ. 1 (2001), 387-404.

6. Grafakos, L., Modern Fourier Analysis, Springer, Berlin, 2009.

7. Grisvard, P., Elliptic Problems in Nonsmooth Domains, Pitman Publishing, Marshfield, MA, 1985.

8. Holst, M., Nagy, G. and Tsogtgerel, G., Rough solutions of the Einstein constraints on closed manifolds without near-CMC conditions, Commun. Math. Phys. 288 (2009), 547-613. arXiv:0712.0798 [gr-qc].

9. Runst, T. and Sickel, W., Sobolev Spaces of Fractional Order, Nemytskij Operators, and Nonlinear Partial Differential Equations, de Gruyter, Berlin, 1996.

10. Stakgold, I. and Holst, M., Green's Functions and Boundary Value Problems, 3rd ed., Wiley, New York, NY, 2011. 888 pages.

11. Triebel, H., Interpolation theory, function spaces, differential operators, NorthHolland Publishing Company, 1977.

12. Zolesio, J., Multiplication dans les espaces de Besov, Proc. R. Soc. Edinb. A 78 (1977), 113-117. 
A. Behzadan

Department of Mathematics and Statistics California State University Sacramento

CA US-95819 Sacramento

U.S.A.

a.behzadan@csus.edu

Received March 1, 2021

in revised form May 18, 2021

\author{
M. Holst \\ Department of Mathematics \\ University of California San Diego \\ CA US-92093 La Jolla \\ U.S.A. \\ mholst@math.ucsd.edu
}

